\begin{tabular}{|c|c|}
\hline \multicolumn{2}{|c|}{ Statistica Sinica Preprint $\quad$ No: SS-2021-0118 } \\
\hline Title & $\begin{array}{l}\text { Regression Analysis of Spatially Correlated Event } \\
\text { Durations With Missing Origins Annotated by } \\
\text { Longitudinal Measures }\end{array}$ \\
\hline Manuscript ID & SS-2021-0118 \\
\hline URL & http://www.stat.sinica.edu.tw/statistica/ \\
\hline DOI & $10.5705 /$ ss.202021.0118 \\
\hline Complete List of Authors & $\begin{array}{l}\text { Yi Xiong, } \\
\text { W. John Braun, } \\
\text { Thierry Duchesne and } \\
\text { X. Joan } \mathrm{Hu}\end{array}$ \\
\hline Corresponding Author & X. Joan $\mathrm{Hu}$ \\
\hline E-mail & joanh@stat.sfu.ca \\
\hline
\end{tabular}




\title{
REGRESSION ANALYSIS OF SPATIALLY CORRELATED EVENT DURATIONS WITH MISSING ORIGINS ANNOTATED BY LONGITUDINAL MEASURES
}

\author{
Yi Xiong, W. John Braun, Thierry Duchesne, and X. Joan Hu \\ Fred Hutchinson Cancer Research Center, Simon Fraser University, \\ University of British Columbia-Okanagan, and Université Laval
}

\begin{abstract}
This paper is concerned with event durations in situations where the study units may be spatially correlated and the time origins of the events are missing. We develop regression models based on the partly observed durations with the aid of available longitudinal information. The first-hitting-time model (e.g. Lee and Whitmore, 2006) is employed to link the data of event durations and the associated longitudinal measures with shared random effects. We present procedures for estimating the model parameters and an induced estimator of the conditional distribution of the event duration. We apply the EM algorithm and Monte Carlo methods to compute the proposed estimators. We establish consistency and asymptotic normality of the estimators, and present their variance estimation. The proposed approach is illustrated with a collection of wildfire records from Alberta, Canada. Its performance is examined numerically and compared with two competitors via simulation.
\end{abstract}

Key words and phrases: Asymptomatic event, first hitting time, EM algorithm and Monte Carlo method, joint modelling, mixed effects model

\section{Introduction}

Many research studies are primarily interested in relating an event duration with possible covariates for purposes of inference and/or prediction. We are particularly concerned with regression based on event-duration observations with missing time 
origins. Such observations arise in infectious disease research, wildfire management, and other areas. For example, many studies are concerned with the incubation period of coronavirus disease 2019 (COVID-19); see, for example, Qin et al. (2020). The incubation period is defined as the duration between the infection time and the onset time of symptoms. However, the exact infection time is unknown and the time based on the individual's recollection is used as a proxy of the true infection time. As another example, the interval from the start time of a wildfire to when suppression activities (the so-called time of initial attack) begin is sometimes used as a gauge of fire management effectiveness and provides important information for predicting the development of the fire. Once a fire is detected and reported, resources such as a fire crew or an airtanker are allocated to suppress the blaze (e.g. Martell, 2007; Morin, 2014). Because fires are not always detectable until they reach a certain size, the start time of a wildfire is always unknown; instead, the time of report is often used as a surrogate when assessing the time to initial attack. Although various regression methods have been developed to evaluate the association of a single variable with its covariates, most regression approaches are not directly applicable to such observations.

Xiong, Braun, and $\mathrm{Hu}(2021)$ propose an approach to tackle the problem of missing time origins via longitudinal measures of an associated quantity. The wellknown empirical distribution function of the event duration based on independent and identically distributed (i.i.d.) observations is adapted for use as an estimator of the marginal distribution using partly observed durations caused by missing time origins. In the context of the wildfire management example, the duration of interest, which we refer to as ISA duration, is the interval between the time a wildfire starts and the initial attack time. Xiong, Braun, and $\mathrm{Hu}(2021)$ apply the procedure for 
estimating the distribution of the ISA duration in each subregion. The estimated distributions appeared to vary regionally and the ISA durations depend on weather variables, such as relative humidity, temperature and wind speed. Fires that are in close proximity would also be expected to be burning similar types of vegetation, in terms of both the understory and the canopy. Topography also plays a role, since fires tend to burn faster up a slope. Thus the ISA durations are potentially spatially correlated. These considerations have partially motivated the research presented in this paper. We are primarily concerned with regression analysis in situations where the response variable is an event duration and the available observations have missing time origins.

When longitudinal data are available together with time-to-event data, a joint model is often considered in the existing literature. A typical model setting is a linear or nonlinear mixed-effects model for the longitudinal measures and a semiparametric or parametric regression model for the time-to-event data, with the two models sharing some random effects or variables. Estimation methods for such joint models have received much attention (e.g. Tseng, Hsieh, and Wang, 2005; $\mathrm{Wu}$, Liu, and $\mathrm{Hu}$, 2010; Wulfsohn and Tsiatis, 1997); detailed reviews of recent work can be found in Furgal, Sen, and Taylor (2019) and Papageorgiou et al. (2019). However, no existing methods can be applied when the time origin is missing for both the event process and the longitudinal process.

This paper assumes the ISA duration follows a semiparametric accelerated failure time (AFT) regression model. It accounts for the potential spatial correlation of the duration times and adjusts for their missing time origins via available longitudinal data. We specify the regression function in the AFT model as a linear combination of some or all of the predictors together with nonparametric terms for 
predictors that are nonlinearly related to the response. We follow Xiong, Braun, and $\mathrm{Hu}$ (2021) in overcoming the challenge posed by the missing time origin by employing associated longitudinal measures and specifying the longitudinal process as a Brownian motion with random drift. Longitudinal measures provide us with information about the missing time origin and assist the inference on the unknown duration. This approach also allows us to link the longitudinal process and the regression model through shared random effects to accommodate spatial correlation among individuals. Our approach may be viewed as an extension of the BuckleyJames estimator (Buckley and James, 1979), which adapts the classical least squares estimation (LSE) to right-censored observations on the response. A similar effort is seen in the interesting work of Ning, Qin, and Shen (2011).

The methodology of threshold regression (TR) provides an alternative to the joint modelling of longitudinal data and time-to-event data. In TR, the event durations are interpreted as the first hitting times (FHTs) of a boundary or threshold state crossed by sample paths of a longitudinal process (Lee and Whitmore, 2006). For example, in HIV studies, CD4 counts are commonly used markers for the health status of HIV-infected individuals, and the time at which AIDS develops can be viewed as the time when the CD4 process first reaches 200 (Doksum and Normand, 1995). As another example, Xiong, Braun, and $\mathrm{Hu}$ (2021) estimate the distribution of the ISA duration by defining the FHT to be the reporting delay, which is the time for the burnt area to reach the reported size.

Spatial correlation adds another layer of complexity to the analysis. Frailty models are commonly employed with spatially correlated time-to-event data (e.g. Banerjee, Wall, and Carlin, 2003; Li and Ryan, 2002; Motarjem, Mohammadzadeh, and Abyar, 2017). As in frailty models, we embed the spatial correlation in the 
random effects.

To facilitate the development of the proposed estimation procedure, we begin by assuming independence among the individuals. The parameters of the longitudinal process are estimated based on the likelihood, and the regression function in the AFT model is estimated by an adaptation of LSE integrated with kernel smoothing. We also propose an estimator of the conditional distribution of the duration given covariates in the presence of a missing time origin. We then extend the procedure to spatially correlated study units. The approach is illustrated for a dataset of wildfires in Alberta, Canada.

The rest of the paper is organized as follows. In Section 2, we describe the regression model of the duration along with the stochastic model for longitudinal measures of the area burned over time. In Section 3, we present the estimation procedure for i.i.d. observations and its extension to spatial correlation, and we derive their asymptotic properties. Section 4 applies our approach to the analysis of wildfire records, and Section 5 presents simulation studies to examine its finitesample performance. Concluding remarks are made in Section 6.

\section{Notation and Modelling}

\section{$2.1 \quad$ Notation}

We follow the notation introduced in Xiong, Braun, and $\mathrm{Hu}$ (2021). Consider a wildfire with its start time, report time, and initial attack time denoted by $T_{S}, T_{R}, T_{F}$. The aforementioned ISA duration is then $L=T_{F}-T_{S}$. Let $A(a)$ be the burnt area of a fire at the elapsed time $a$ since it starts: $A(0)=0$ and $A(S)=B$ are the respective burnt areas at the start time and report time. Here $S=T_{R}-T_{S}$ represents the fire's elapsed time till the report time. Note that $L=S+L^{*}$ with $L^{*}=T_{F}-T_{R}$. 
While $L^{*}$ may be observed, the reporting delay $S$ is usually unavailable and thus so is $L$. Further, let $A(L)-A(S)=D$ be the increased burnt area over the interval $\left(T_{R}, T_{F}\right)$

We denote the location where a fire is detected by $\boldsymbol{\omega}=\left(\omega_{1}, \omega_{2}\right)^{\prime}$, and a vector of environmental and spatial factors (e.g. wind speed, fuel type, and region) associated with the fire at the report time by $\mathbf{X}$. Figure 1 presents a progression description of two hypothetical wildfires with the notation. The solid curve in each plot represents the burnt area over time of a fire that is subject to suppression after detection.

- Figure 1 is here.

Suppose that a collection of records on $i=1, \ldots, n$ wildfires is available, denoted by

$$
\mathcal{O}=\bigcup_{i=1}^{n} \mathcal{O}_{i}=\left\{\left(T_{R i}, T_{F i}, B_{i}, D_{i}, \mathbf{X}_{i}, \boldsymbol{\omega}_{i}\right): i=1,2, \cdots, n\right\}
$$

Our primary statistical interest is in estimating the conditional distribution of the ISA duration $L$ given the covariate vector $\mathbf{X}, P(L \leq t \mid \mathbf{X}=\mathbf{x})$, with the available data. The estimated $P(L \leq t \mid \mathbf{X}=\mathbf{x})$ may reveal how the ISA duration $L=S+L^{*}$ is associated with the covariate vector $\mathbf{X}$, and can be applied for prediction.

Note that the available data $\mathcal{O}=\bigcup_{i=1}^{n} \mathcal{O}_{i}$ in 2.1 include observations on $L^{*}=$ $T_{F}-T_{R}$ rather than on $L$. The burnt area records may provide information about the reporting delay $S$ and thus the ISA duration $L$. In the following subsections, we present our modeling of the conditional distribution $P(L \leq t \mid \mathbf{X}=\mathbf{x})$ along with the component models that link the burnt areas to the reporting delay.

\subsection{Longitudinal Model for Repeated Measures}

We first use a time-indexed stochastic process $A_{i}(\cdot)$ to model the longitudinal measures associated with each study unit $i$. In the wildfire application, they are burnt 
areas of fire $i$ over time. We assume that, conditional on the covariates $\mathbf{X}_{i}$ and random effects $\left(\delta_{1 i}, \delta_{2 i}\right)$, the area burned at time $u$ follows the mixed effect model:

$$
A_{i}(u)=\nu_{i} u+\sigma W_{i}(u), i=1,2, \cdots, n,
$$

where $\nu_{i}=\nu \exp \left\{\delta_{1 i}+\mathbf{X}_{i}^{\prime} \boldsymbol{\gamma}+X_{i}^{*} \delta_{2 i}\right\}$ with constants $\nu>0, \sigma>0$ and $W_{i}(\cdot)$ is the standard Wiener process. Note we only model the longitudinal burnt areas before initial attack in the wildfire application. As presented in Figure 1, the dashed curve in each plot shows the expected trajectory of the fire's burnt area if it had continued to burn without any suppression or intervention. Prior to initial attack, the dashed curve coincides with the solid curve and the growth of the fire's burnt areas can be approximated as assumed in $(2.2)$.

Here, $\delta_{1 i}$ together with $\nu$ yields the random intercept term $\log \nu+\delta_{1 i}$ of $\log \nu_{i}, X_{i}^{*}$ is one of the components of the covariate vector $\mathbf{X}_{i}$, and $\delta_{2 i}$ is its (random) coefficient. This model allows the drifts $\nu_{i}$ to accommodate individual-specific covariate effects. We assume that $\delta_{1 i}$ and $\delta_{2 i}$ are independent of each other and independent of $W_{i}(\cdot)$, for $i=1, \cdots, n$. Furthermore, $\boldsymbol{\delta}_{1}=\left(\delta_{11}, \cdots, \delta_{1 n}\right)^{\prime}$ and $\boldsymbol{\delta}_{2}=\left(\delta_{21}, \cdots, \delta_{2 n}\right)^{\prime}$ are assumed to follow the distributions $\operatorname{MVN}\left(0, \Sigma_{1}\right)$ and $M V N\left(0, \Sigma_{2}\right)$, respectively. We consider the following two specifications for the covariance matrices $\Sigma_{1}, \Sigma_{2}$ :

- Independent Study Units. Let $\Sigma_{1}=\psi_{1}^{2} I_{n \times n}$ and $\Sigma_{2}=\psi_{2}^{2} I_{n \times n}$, where $I_{n \times n}$ is the identity matrix of size $n$. Then $\delta_{1 i} \sim N\left(0, \psi_{1}^{2}\right)$ and $\delta_{2 i} \sim N\left(0, \psi_{2}^{2}\right)$ for $i=1,2, \cdots, n$. Denote the density functions by $\phi_{1}\left(\cdot ; \psi_{1}\right)$ and $\phi_{2}\left(\cdot ; \psi_{2}\right)$, respectively.

- Spatially Correlated Study Units. Define the $(i, j)$ element of $\Sigma_{1}$ and $\Sigma_{2}$ as 


$$
\left(\Sigma_{k}\right)_{i j}=\psi_{k}^{2} \exp \left(-\left\|\boldsymbol{\omega}_{\boldsymbol{i}}-\boldsymbol{\omega}_{\boldsymbol{j}}\right\| / \rho_{k}\right) \text { for } k=1,2
$$

where $\|\cdot\|$ is the Euclidean norm. In the rest of this paper, the covariance matrices $\Sigma_{k}$ are denoted by $\Sigma\left(\psi_{k}, \rho_{k}\right)$ for $k=1,2$. The spatial correlation is assumed to decay as the geographic distance increases, and $\rho_{k}=0$ corresponds to the situation where the $\delta_{k i}^{\prime} s$ are independent with variance $\psi_{k}$.

\subsection{Regression Models for Event Duration}

We consider the regression model of the transformed ISA durations $Y_{i}=\log L_{i}$ as follows:

$$
Y_{i}=h\left(\mathbf{X}_{i}, \delta_{1 i}, \delta_{2 i}\right)+\epsilon_{i}
$$

where the random errors $\epsilon_{i}$ are independent of the covariates $\mathbf{X}_{i}$ and the random effects $\left(\delta_{1 i}, \delta_{2 i}\right)$, and follow an unspecified distribution function $F_{\epsilon}(\cdot)$ with $\mathrm{E}\left[\epsilon_{i}\right]=$ 0 . The random intercept $\delta_{1 i}$ and the random slope $\delta_{2 i}$ are shared terms in the longitudinal model 2.2 . When study units are spatially correlated, the vector $\boldsymbol{\delta}_{k}=\left(\delta_{k 1}, \cdots, \delta_{k n}\right)^{\prime}$ for $k=1,2$ follow the multivariate normal distribution with the covariance matrix specified in (2.3). In the case where study units are assumed to be independent, $\delta_{1 i} \stackrel{i . i . d}{\sim} N\left(0, \psi_{1}^{2}\right)$ and $\delta_{2 i} \stackrel{i . i . d}{\sim} N\left(0, \psi_{2}^{2}\right)$ for $i=1,2, \cdots, n$. Then model (2.4) reduces to: $Y_{i}=h\left(\mathbf{X}_{i}\right)+\epsilon_{i}$, for $i=1,2, \cdots, n$.

By specifying $h(\cdot)$ as a linear function or a partially linear function, special cases for the model (2.4) with independent and correlated study units are given as below.

- Special cases of Model (2.4) for independent study units:

$$
\begin{aligned}
& \text { LRM-Indpt: } Y_{i}=\beta_{0}+\mathbf{X}_{i}^{\prime} \boldsymbol{\beta}_{1}+\epsilon_{i}, \\
& \text { PLRM-Indpt: }: Y_{i}=\mathbf{X}_{i}^{\dagger^{\prime}} \boldsymbol{\beta}^{\dagger}+h_{0}\left(\mathbf{X}_{i}^{\ddagger}\right)+\epsilon_{i},
\end{aligned}
$$


where LRM and PLRM stand for linear regression model and partially linear regression model, respectively.

- Special cases of Model (2.4) for correlated study units:

$$
\begin{aligned}
& \text { LRM-Corrltd: } Y_{i}=\beta_{0}+\mathbf{X}_{i}^{\prime} \boldsymbol{\beta}_{1}+\delta_{1 i} \beta_{2}+\delta_{2 i} \beta_{3}+\epsilon_{i}, \\
& \text { PLRM-Corrltd: } Y_{i}=\beta_{0}+\mathbf{X}_{i}^{\dagger^{\prime}} \boldsymbol{\beta}_{1}^{\dagger}+\delta_{1 i} \beta_{2}^{\dagger}+\delta_{2 i} \beta_{3}^{\dagger}+h_{0}\left(\mathbf{X}_{i}^{\ddagger}\right)+\epsilon_{i},
\end{aligned}
$$

where $\mathbf{X}_{i}^{\dagger}$ and $\mathbf{X}_{i}^{\ddagger}$ are subsets of the covariates $\mathbf{X}_{i}$.

\subsection{Induced Distribution of First-hitting-time}

Under the Wiener process model (2.2), the reporting delay $S_{i}$ may be viewed as the FHT, i.e. the time when the process $A_{i}(\cdot)$ first reaches the threshold $B_{i}$, that is the area burned at the report time: $S_{i}=\inf \left\{u: u>0, A_{i}(u)>B_{i}\right\}$, which is the same as $\sup \left\{u: u>0, A_{i}(u)<B_{i}\right\}$ almost surely. The FHT $S_{i}$ follows the inverse Gaussian (IG) distribution (e.g. Chhikara and Folks, 1989) with the cumulative distribution function $G\left(u \mid B_{i}, \mathbf{X}_{i} ; \mu_{i}, \lambda_{i}\right)$ being

$$
\Phi\left(\sqrt{\frac{\lambda_{i}}{u}}\left[\frac{u}{\mu_{i}}-1\right]\right)+\exp \left(2 \lambda_{i} / \mu_{i}\right) \Phi\left(-\sqrt{\frac{\lambda_{i}}{u}}\left[\frac{u}{\mu_{i}}+1\right]\right)
$$

where $\mu_{i}=B_{i} / \nu e^{\mathbf{X}^{\prime}{ }_{i} \boldsymbol{\gamma}+X_{i}^{*} \delta_{2 i}+\delta_{1 i}}, \lambda_{i}=B_{i}^{2} / \sigma^{2}$, and $\Phi(\cdot)$ is the cumulative distribution function of the standard normal distribution. We denote the cumulative distribution of $S_{i}$ by $G\left(u \mid B_{i}, \mathbf{X}_{i}, \delta_{1 i}, \delta_{2 i}, \nu, \sigma, \gamma\right)$.

On the other hand, the unobserved reporting delay $S_{i}$ is a portion of the event duration $L_{i}$, the response variable in the desired regression analysis. By making use of the related longitudinal measures and the induced distribution of first hitting time, we can overcome the inherent difficulty of the unobserved duration in the regression analysis. Moreover, $\left(\delta_{1 i}, \delta_{2 i}\right)$, the shared random effects/frailty variables, are used to connect the event duration and longitudinal measures and to capture the spatial correlation among individuals. 


\section{Estimation in the Presence of Missing Time Origins}

This section presents the estimation of the conditional distribution of the event duration $F(t \mid \mathbf{x})=P(L \leq t \mid \mathbf{x})$ in situations where the study units are independent or spatially correlated. We must estimate the regression function $h(\cdot)$ and the distribution of the random error. We start with the estimation procedure with independent units and then adapt the procedure for spatially correlated units.

\subsection{Estimation with Independent Study Units}

The following two assumptions are made throughout this section:

$\operatorname{Assumption~(A1).~}\left\{\left(T_{S i}, T_{R i}, T_{F i}, B_{i}, D_{i}, \mathbf{X}_{i}, \boldsymbol{\omega}_{i}\right): i=1,2, \cdots, n\right\}$ is a collection of i.i.d. realizations of $\left(T_{S}, T_{R}, T_{F}, B, D, \mathbf{X}, \boldsymbol{\omega}\right)$.

Assumption (A2). For $i=1,2, \cdots, n, L_{i}^{*}=T_{F i}-T_{R i}=L_{i}-S_{i}$ and $S_{i}=$ $T_{R i}-T_{S i}$ are conditionally independent given $\left(\delta_{1 i}, \delta_{2 i}\right)$ and $\mathbf{X}_{i}$. In addition, $B_{i}$ and $\left(\delta_{1 i}, \delta_{2 i}\right)$ are conditionally independent given $\mathbf{X}_{i}$.

In the wildfire application, the conditional independence assumption for $S_{i}$ and $L_{i}^{*}$ in (A2) is plausible since the fire agency often assesses a reported fire regarding its spread rate $\left(\nu_{i}\right)$, and then plans for initial attack accordingly. As $\nu_{i}$ is specified depending on $\mathbf{X}_{i}$ and $\left(\delta_{1 i}, \delta_{2 i}\right), L_{i}^{*}$ is likely associated with $S_{i}$ solely through $\mathbf{X}_{i}$ and $\left(\delta_{1 i}, \delta_{2 i}\right)$

\subsubsection{Proposed Estimator for $F(\cdot \mid \mathbf{x})$}

From regression models LRM-Indpt 2.4a and PLRM-Indpt 2.4b), $P(L \leq t \mid \mathbf{X}=\mathbf{x})=$ $F_{\epsilon}(\log (t)-h(\mathbf{x}))$. When all the durations $L_{i}$ are observed, $F_{\epsilon}(\cdot)$ can be estimated by the empirical function $F_{\epsilon, n}(\zeta)=n^{-1} \sum_{i=1}^{n} I\left(\epsilon_{i} \leq \zeta\right)$ and it can be also written as $n^{-1} \sum_{i=1}^{n} I\left(S_{i} \leq e^{\zeta+h\left(\mathbf{X}_{i}\right)}-L_{i}^{*}\right)$. 
Note that $\mathrm{E}\left\{I\left(\epsilon_{i} \leq \zeta\right) \mid \mathcal{O}_{i}\right\}=P\left(\epsilon_{i} \leq \zeta \mid \mathcal{O}_{i}\right)=P\left(S_{i} \leq e^{\zeta+h\left(\mathbf{X}_{i}\right)}-L_{i}^{*} \mid \mathcal{O}_{i}\right)$. With Model (2.2) and Assumption (A2), $P\left(S_{i} \leq e^{\zeta+h\left(\mathbf{X}_{i}\right)}-L_{i}^{*} \mid \mathcal{O}_{i}\right)$ is

$$
\iint G\left(e^{\zeta+h\left(\mathbf{X}_{i}\right)}-L_{i}^{*} \mid B_{i}, \mathbf{X}_{i}, \delta_{1}, \delta_{2} ; \nu, \sigma, \gamma\right) \phi_{1}\left(\delta_{1} \mid \mathcal{O}_{i} ; \boldsymbol{\theta}\right) \phi_{2}\left(\delta_{2} \mid \mathcal{O}_{i} ; \boldsymbol{\theta}\right) \mathrm{d} \delta_{1} \mathrm{~d} \delta_{2}
$$

where $\boldsymbol{\theta}=\left(\nu, \sigma, \gamma, \psi_{1}, \psi_{2}\right)^{\prime}$, and $\phi_{1}\left(\cdot \mid \mathcal{O}_{i} ; \boldsymbol{\theta}\right)$ and $\phi_{2}\left(\cdot \mid \mathcal{O}_{i} ; \boldsymbol{\theta}\right)$ are the conditional distributions of $\delta_{1}$ and $\delta_{2}$ given the observed data $\mathcal{O}_{i}$. If $\boldsymbol{\theta}$ and $h(\cdot)$ are known, we can estimate $F_{\epsilon}(\zeta)$ by $\tilde{F}_{\epsilon, n}(\zeta ; \boldsymbol{\theta}, h(\cdot))=n^{-1} \sum_{i=1}^{n} P\left(S_{i} \leq e^{\zeta+h\left(\mathbf{X}_{i}\right)}-L_{i}^{*} \mid \mathcal{O}_{i}\right)$. Thus, $P(L \leq t \mid \mathbf{X}=\mathbf{x})=F_{\epsilon}(\log t-h(\mathbf{x}))$ can be estimated by $\tilde{F}_{n}(t \mid \mathbf{x} ; \boldsymbol{\theta}, h(\cdot))=$ $\tilde{F}_{\epsilon, n}(\log t-h(\mathbf{x}) ; \boldsymbol{\theta}, h(\cdot))$. Upon substitution of the estimators for $\boldsymbol{\theta}$ and $h(\cdot)$, we obtain the following estimator for $F(t \mid \mathbf{x})$ :

$$
\hat{F}_{n}(t \mid \mathbf{x})=\tilde{F}_{n}(t \mid \mathbf{x} ; \hat{\boldsymbol{\theta}}, \hat{h}(\cdot))=\tilde{F}_{\epsilon, n}(\log t-h(\mathbf{x}) ; \hat{\boldsymbol{\theta}}, \hat{h}(\cdot)) .
$$

We present the procedure for estimating $\boldsymbol{\theta}$ and $h(\cdot)$ in the next subsection.

\subsubsection{Estimation Procedure for $\boldsymbol{\theta}$ and $h(\cdot)$}

We now present a two-stage procedure for estimating $\boldsymbol{\theta}$ and $h(\cdot)$ under the independence assumption.

Stage I: Estimation of $\boldsymbol{\theta}$ Let $\boldsymbol{\theta}=\left(\nu, \sigma, \gamma, \psi_{1}, \psi_{2}\right)$. Conditional on the covariates $\mathbf{X}$, the log-likelihood function with the observed data (2.1) is

$$
l_{n}(\boldsymbol{\theta})=\sum_{i=1}^{n} \log \iiint\left\{L_{o b s, i \mid S, \delta_{1}, \delta_{2}, \mathbf{X}_{i}}\right\} \mathrm{d}\left[S, \delta_{1}, \delta_{2} \mid \mathbf{X}_{i}\right]
$$

where $L_{o b s, i \mid S, \delta_{1}, \delta_{2}, \mathbf{X}_{i}}=\left[D_{i} \mid B_{i}, L_{i}^{*}, \delta_{1}, \delta_{2}, \mathbf{X}_{i}\right]\left[B_{i} \mid S, \delta_{1}, \delta_{2}, \mathbf{X}_{i}\right]$.

Let the "full dataset" be the observed data 2.1) augmented by $\underline{S}=\left\{S_{i}, i=\right.$ 
$1,2, \cdots, n\}, \underline{\delta_{1}}=\left\{\delta_{1 i}, i=1,2, \cdots, n\right\}$, and $\underline{\delta_{2}}=\left\{\delta_{2 i}, i=1,2, \cdots, n\right\}$. The loglikelihood function conditional on the covariates $\mathbf{X}$ with the full data is given by $l_{F}\left(\boldsymbol{\theta} \mid\right.$ Observed-data, $\left.\underline{S}, \underline{\delta_{1}}, \underline{\delta_{2}}\right)=l_{F_{1}}\left(\nu, \sigma, \gamma \mid \underline{S}, \underline{\delta_{1}}, \underline{\delta_{2}}\right)+l_{F_{2}}\left(\boldsymbol{\theta} ; \underline{S}, \underline{\delta_{1}}, \underline{\delta_{2}}\right)$, where $l_{F_{1}}\left(\nu, \sigma, \gamma \mid \underline{S}, \underline{\delta_{1}}, \underline{\delta_{2}}\right)$ is

$$
-n \log \sigma^{2}-\sum_{i=1}^{n} \frac{\left(D_{i}-\nu e^{\mathbf{X}_{i}^{\prime} \boldsymbol{\gamma}+X_{i}^{*} \delta_{2 i}+\delta_{1 i}} L_{i}^{*}\right)^{2}}{2 \sigma^{2} L_{i}^{*}}-\sum_{i=1}^{n} \frac{\left(B_{i}-\nu e^{\mathbf{X}_{i}^{\prime} \boldsymbol{\gamma}+X_{i}^{*} \delta_{2 i}+\delta_{1 i}} S_{i}\right)^{2}}{2 \sigma^{2} S_{i}},
$$

and

$$
l_{F_{2}}\left(\boldsymbol{\theta} ; \underline{S}, \underline{\delta_{1}}, \underline{\delta_{2}}\right)=\sum_{i=1}^{n} \log \left[S_{i} \mid \delta_{1 i}, \delta_{2 i}, \mathbf{X}_{i}\right]+\sum_{i=1}^{n} \log \phi_{1}\left(\delta_{1 i} ; \psi_{1}\right)+\sum_{i=1}^{n} \log \phi_{2}\left(\delta_{2 i} ; \psi_{2}\right) .
$$

The following Monte-Carlo EM algorithm is used to compute the MLE $\hat{\boldsymbol{\theta}}_{n}$. 


\section{Algorithm A}

1: Assume that we have the estimate $\boldsymbol{\theta}^{(m)}$ at the $m$ th iteration $(m \geq 0)$, with $\boldsymbol{\theta}^{(0)}$ for the initial value.

2: repeat

3: $\quad$ E-step. Approximate $Q\left(\boldsymbol{\theta}, \boldsymbol{\theta}^{(m)}\right)=\mathrm{E}\left\{l_{F}\left(\boldsymbol{\theta} \mid\right.\right.$ Observed-data, $\left.\left.\underline{S}, \underline{\delta_{1}}, \underline{\delta_{2}}\right) \mid \mathcal{O} ; \boldsymbol{\theta}^{(m)}\right\}$ via the sample mean $\frac{1}{J} \sum_{j=1}^{J} l_{F}\left(\boldsymbol{\theta} \mid\right.$ Observed-data, $\left.\underline{S}^{(j)}, \underline{\delta}^{(j)}, \underline{\delta}^{(j)}\right)$, which is given by

$$
\frac{1}{J} \sum_{j=1}^{J} l_{F_{1}}\left(\nu, \sigma, \gamma \mid \underline{S}^{(j)}, \underline{\delta}^{(j)}, \underline{\delta}^{(j)}\right)+\frac{1}{J} \sum_{j=1}^{J} l_{F_{2}}\left(\boldsymbol{\theta} ; \underline{S}^{(j)}, \underline{\delta}^{(j)}, \underline{\delta}^{(j)}\right),
$$

where for $j=1, \cdots, J$, and $\left(\underline{S}^{(j)}, \underline{\delta}_{1}^{(j)}, \underline{\delta}_{2}{ }^{(j)}\right)$ is generated from the conditional distribution given the observed data $\left[\underline{S}, \delta_{1}, \delta_{2} \mid \mathcal{O} ; \boldsymbol{\theta}^{(m)}\right]$. This distribution can also be written as $\left[\underline{S} \mid \underline{\delta_{1}}, \underline{\delta_{2}}, \mathcal{O} ; \boldsymbol{\theta}^{(m)}\right]\left[\underline{\delta_{1}}, \underline{\delta_{2}} \mid \mathcal{O} ; \boldsymbol{\theta}^{(m)}\right]$.

- $\quad$ E-step (i). Generate $\left(\delta_{1 i}^{(j)}, \delta_{2 i}^{(j)}\right)^{\prime}$ from $\phi_{i}^{(m+1)}\left(\delta_{1}, \delta_{2} \mid \mathcal{O}_{i} ; \boldsymbol{\theta}^{(m)}\right)$, which is the conditional distribution of $\left(\delta_{1}, \delta_{2}\right)$ given the observed data with the current parameter estimate $\boldsymbol{\theta}^{(m)}$,

$\frac{L_{o b s, i \mid \delta_{1}, \delta_{2}, \mathbf{X}_{i}}\left(\nu^{(m)}, \sigma^{(m)}, \gamma^{(m)} ; \delta_{1}, \delta_{2}\right) \phi_{1}\left(\delta_{1} ; \psi_{1}^{(m)}\right) \phi_{2}\left(\delta_{2} ; \psi_{2}^{(m)}\right)}{\iint L_{o b s, i \mid \delta_{1}, \delta_{2}, \mathbf{X}_{i}}\left(\nu^{(m)}, \sigma^{(m)}, \gamma^{(m)} ; \delta\right) \phi_{1}\left(\delta_{1} ; \psi_{1}^{(m)}\right) \phi_{2}\left(\delta_{2} ; \psi_{2}^{(m)}\right) \mathrm{d} \delta_{1} \mathrm{~d} \delta_{2}}$,

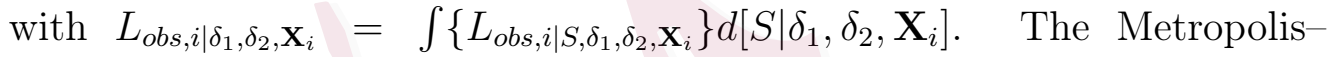
Hastings algorithm (Hastings, 1970, Metropolis et al. 1953) can be applied to generate $\left(\delta_{1 i}^{(j)}, \delta_{2 i}^{(j)}\right)$ with the proposed distribution being $\left(\delta_{1 i}^{(n e w)}, \delta_{2 i}^{(n e w)}\right)^{\prime} \sim \operatorname{MVN}\left(\left(\delta_{1 i}^{(\text {old })}, \delta_{2 i}^{(\text {old })}\right)^{\prime}, 0.5^{2} I_{2 \times 2}\right)$.

- $\quad$-step (ii). Under Assumption (A1), we can generate $S_{i}^{(j)}$, the $i^{\text {th }}$ component of $\underline{S}^{(j)}$, independently from the conditional distribution of $S_{i}$ given the observed data with $\boldsymbol{\theta}^{(m)}$ and $\delta_{1 i}^{(j)}, \delta_{2 i}^{(j)}$. This distribution, $\left[S_{i} \mid \delta_{1 i}, \delta_{2 i}, \mathcal{O}_{i} ; \boldsymbol{\theta}^{(m)}\right]$, is in fact the IG distribution given in 2.5 with $\nu=\nu^{(m)}, \sigma=\sigma^{(m)}, \gamma=\gamma^{(m)}$.

4: $\quad$-step. Obtain the updated $\boldsymbol{\theta}^{(m+1)}$ by maximizing (3.9).

5: until $\left\{\boldsymbol{\theta}^{(m)}: m=1,2, \cdots\right\}$ converges.

Because $\left[S_{i} \mid \delta_{1 i}, \delta_{2 i}, \mathbf{X}_{i}\right]$ in $l_{F_{2}}\left(\boldsymbol{\theta} ; \underline{S}, \underline{\delta_{1}}, \underline{\delta_{2}}\right)$ does not have much additional information on the parameters $\nu, \sigma, \gamma$, the objective function $l_{F}\left(\boldsymbol{\theta} \mid\right.$ Observed-data, $\left.\underline{S}, \underline{\delta_{1}}, \underline{\delta_{2}}\right)$ can be replaced by $l_{F_{1}}\left(\nu, \sigma, \gamma \mid \underline{S}, \underline{\delta_{1}}, \underline{\delta_{2}}\right)+\log \phi_{1}\left(\psi_{1} ; \underline{\delta_{1}}\right)+\log \phi_{2}\left(\psi_{2} ; \underline{\delta_{2}}\right)$. This maximization procedure leads to $\tilde{\boldsymbol{\theta}}_{n}$, a close approximation to the MLE $\hat{\boldsymbol{\theta}}_{n}$. Therefore, the $M$-step can be implemented by solving $\sum_{j=1}^{J} \frac{\partial l_{F_{1}}}{\partial(\nu, \sigma, \gamma)}\left(\nu, \sigma, \gamma \mid \underline{S}^{(j)}, \underline{\delta}^{(j)}, \underline{\delta}^{(j)}\right) / J=0$, $\sum_{j=1}^{J} \sum_{i=1}^{n} \frac{\partial \log \phi_{1}}{\partial \psi_{1}}\left(\delta_{1 i}^{(j)} ; \psi_{1}\right) / J=0$ and $\sum_{j=1}^{J} \sum_{i=1}^{n} \frac{\partial \log \phi_{2}}{\partial \psi_{2}}\left(\delta_{2 i}^{(j)} ; \psi_{2}\right) / J=0$. This al- 
gorithm results in $\tilde{\boldsymbol{\theta}}_{n}$, a close approximation to the MLE $\hat{\boldsymbol{\theta}}_{n}$.

Stage II: Estimation of $h(\cdot)$ Given that Model LRM-Indpt 2.4a uses a linear function of the covariates to approximate $h(\cdot)$, the estimator of $h(\cdot)$ only requires estimation of the parameter $\boldsymbol{\beta}=\left(\beta_{0}, \boldsymbol{\beta}_{1}\right)$. If all the durations $L_{i}$ were observed, a consistent estimator for $\boldsymbol{\beta}$ would be the least-squares estimator $\hat{\boldsymbol{\beta}}_{L S E}=\left(\mathbb{X}^{\prime} \mathbb{X}\right)^{-1} \mathbb{X}^{\prime} \mathbf{Y}$ where $\mathbf{Y}=\left(\log L_{1}, \cdots, \log L_{n}\right)^{\prime}$ and $\mathbb{X}$ is a matrix with the $i^{\text {th }}$ row being $\mathbf{X}_{i}=$ $\left(1, \mathbf{X}_{i}^{\prime}\right)^{\prime}$. Although $L_{i}$ is not available in the data, we have $L_{i}=S_{i}+L_{i}^{*}$ with $L_{i}^{*}$ observed and the distribution of $S_{i}$ conditional on the observed data being the IG distribution. We then consider an estimator, $\tilde{\boldsymbol{\beta}}(\boldsymbol{\theta})=E\left[\hat{\boldsymbol{\beta}}_{L S E} \mid \mathbf{O} ; \boldsymbol{\theta}\right]$. We replace $\boldsymbol{\theta}$ with the estimator $\hat{\boldsymbol{\theta}}_{n}$ from Algorithm A to obtain the estimator $\tilde{\boldsymbol{\beta}}\left(\hat{\boldsymbol{\theta}}_{n}\right)=$ $\mathrm{E}\left[\hat{\boldsymbol{\beta}}_{L S E} \mid \mathcal{O} ; \hat{\boldsymbol{\theta}}_{\boldsymbol{n}}\right]$, denoted $\hat{\boldsymbol{\beta}}_{n}$. The following algorithm, based on the Monte-Carlo method, is used to compute $\hat{\boldsymbol{\beta}}_{n}$.

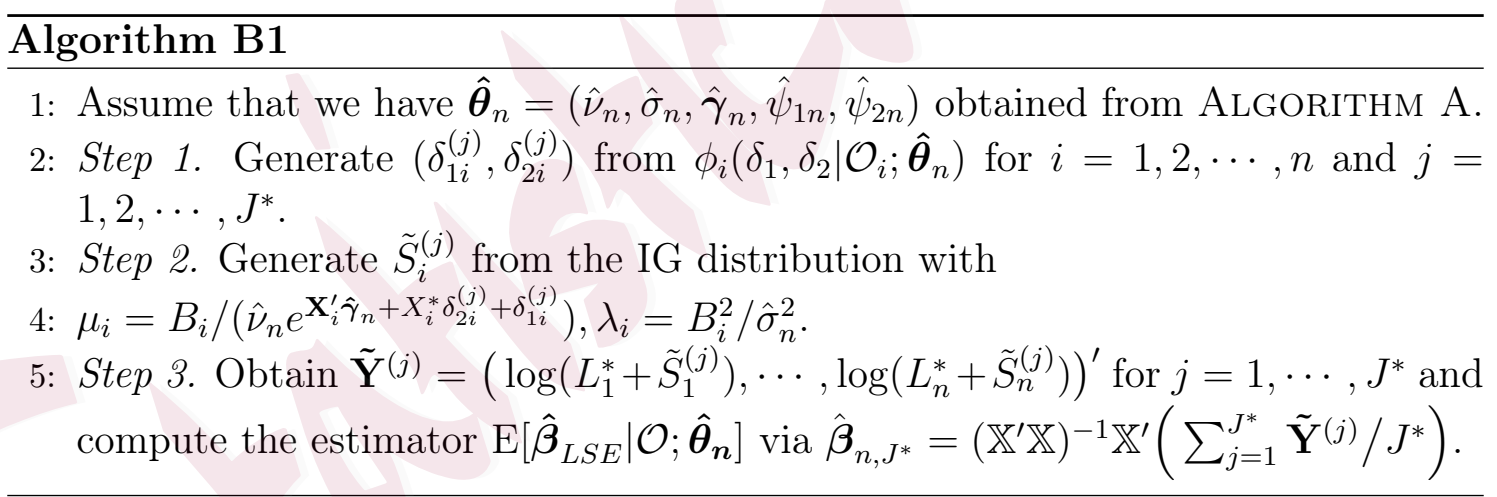

The output of this algorithm, $\hat{\boldsymbol{\beta}}_{n, J^{*}}$ is a close approximation to the estimator $\hat{\boldsymbol{\beta}}_{n}$ when $J^{*}$ is sufficiently large.

Under Model PLRM-Indpt (2.4b), estimation of $h(\cdot)$ requires estimation of both the parametric component, i.e. the parameter $\boldsymbol{\beta}^{\dagger}$, and the nonparametric function $h_{0}(\cdot)$. When all the durations are available, we can follow Speckman (1988) and derive estimators of the parametric and nonparametric components using LSE with kernel smoothing: $\hat{\boldsymbol{\beta}}_{\text {speck }}^{\dagger}=\left(\breve{\mathbb{X}}_{\mathbf{d}}^{\dagger} \breve{\mathbb{X}}_{\mathbf{d}}^{\dagger}\right)^{-1} \breve{\mathbb{X}}_{\mathbf{d}}^{\dagger} \breve{\mathbf{Y}}_{\mathbf{d}}$, and $\hat{h}_{0, \text { speck }}\left(\mathbf{x}^{\ddagger}\right)=$ 
$\frac{\sum_{i=1}^{n} K_{\mathbf{d}}\left(\mathbf{x}^{\ddagger}-\mathbf{X}_{i}^{\ddagger}\right)\left(Y_{i}-\mathbf{X}_{i}^{\dagger^{\prime}} \hat{\boldsymbol{\beta}}_{\text {speck }}^{\dagger}\right)}{\sum_{i=1}^{n} K_{\mathbf{d}}\left(\mathbf{x}^{\ddagger}-\mathbf{X}_{i}^{\ddagger}\right)}$, where $\mathbb{X}^{\dagger}$ is a matrix with the $i^{\text {th }}$ row being $\mathbf{X}_{i}^{\dagger^{\prime}}, \breve{\mathbb{X}}_{\mathbf{d}}^{\dagger}=$ $\left(\mathbf{I}-\mathbf{H}_{\mathbf{d}}\right) \mathbb{X}^{\dagger}$ and $\breve{\mathbf{Y}}_{\mathbf{d}}=\left(\mathbf{I}-\mathbf{H}_{\mathbf{d}}\right) \mathbf{Y}$. Here, $K_{\mathbf{d}}(\cdot)$ is a kernel function with bandwidth $\mathbf{d}$ and $\mathbf{H}_{\mathbf{d}}$ is a smoothing matrix with the $(i, j)^{t h}$ element being $K_{\mathbf{d}}\left(\mathbf{X}_{i}^{\ddagger}-\right.$ $\left.\mathbf{X}_{j}^{\ddagger}\right) / \sum_{j=1}^{n} K_{\mathbf{d}}\left(\mathbf{X}_{i}^{\ddagger}-\mathbf{X}_{j}^{\ddagger}\right)$. The fitted values $\hat{\mathbf{Y}}=\left(\hat{Y}_{1}, \cdots, \hat{Y}_{n}\right)^{\prime}$ can be obtained by $\hat{\mathbf{Y}}=\left(\mathbf{H}_{\mathbf{d}}+\breve{\mathbb{X}}_{\mathbf{d}}^{\dagger}\left(\breve{\mathbb{X}}_{\mathbf{d}}^{\dagger^{\prime}} \breve{\mathbb{X}}_{\mathbf{d}}^{\dagger}\right)^{-1} \breve{\mathbb{X}}_{\mathbf{d}}^{\dagger^{\prime}}\left(\mathbf{I}-\mathbf{H}_{\mathbf{d}}\right)\right) \mathbf{Y}$. Let $\mathbf{A}_{d}$ be the smoothing matrix calculated with bandwidth $\mathbf{d}$ and $\mathbf{A}_{\mathbf{d}}=\left(\mathbf{H}_{\mathbf{d}}+\breve{\mathbb{X}}_{\mathbf{d}}^{\dagger}\left(\breve{\mathbb{X}}_{\mathbf{d}}^{\dagger} \breve{\mathbb{X}}_{\mathbf{d}}^{\dagger}\right)^{-1} \breve{\mathbb{X}}_{\mathbf{d}}^{\dagger}\left(\mathbf{I}-\mathbf{H}_{\mathbf{d}}\right)\right)$. We use the generalized cross-validation (GCV) criterion (e.g. Loader, 1999) to select the bandwidth. The GCV function is

$$
G C V(\mathbf{d})=\frac{\left\|\left(\mathbf{I}-\mathbf{A}_{\mathbf{d}}\right) \mathbf{Y}\right\|^{2}}{\left[1-n^{-1} \operatorname{trace}\left(\mathbf{A}_{\mathbf{d}}\right)\right]^{2}}
$$

Following the idea of AlgORITHM B1, we can also consider estimators $\hat{\boldsymbol{\beta}}_{n}^{\dagger}=$ $\mathrm{E}\left[\hat{\boldsymbol{\beta}}_{\text {speck }}^{\dagger} \mid \mathcal{O} ; \hat{\boldsymbol{\theta}}_{n}\right]$ and $\hat{h}_{0 n}(\cdot)=\mathrm{E}\left[\hat{h}_{0, \text { speck }}(\cdot) \mid \mathcal{O} ; \hat{\boldsymbol{\theta}}_{n}\right]$ in our situation where the duration is not observed. We can then adapt Step 3 in Algorithm B1 to the PLRM-Indpt 2.4b). This yields the following algorithm.

\section{Algorithm B2}

1: Assume that we have $\hat{\boldsymbol{\theta}}_{n}$ obtained from Algorithm A.

2: Steps 1 and 2. Follow Steps 1 and 2 of Algorithm B1 to generate $\left(\delta_{1 i}^{(j)}, \delta_{2 i}^{(j)}\right)$, and $S_{i}^{(j)}$ for $i=1, \cdots, n$ and $j=1, \cdots, J^{*}$.

3: Step 3. For $j=1,2, \cdots, J^{*}$, obtain the vector $\tilde{\mathbf{Y}}^{(j)}$ with the $i^{\text {th }}$ element being $\tilde{Y}_{i}^{(j)}=\log \left(L_{i}^{*}+\tilde{S}_{i}^{(j)}\right)$.

- Step 3(i) For $j=1, \cdots, J^{*}$, compute the bandwidth $\mathbf{d}^{(j)}$ with $\left\{\tilde{Y}_{i}^{(j)}, i=\right.$ $1, \cdots, n\}$ using the GCV function in 3.10 . Compute the vector $\tilde{\mathbf{Y}}_{\mathbf{d}^{(j)}}^{(j)}=$ $\left(\mathbf{I}-\mathbf{H}_{\mathbf{d}^{(j)}}\right) \tilde{\mathbf{Y}}^{(j)}$, where the smoothing matrix $\mathbf{H}_{\mathbf{d}^{(j)}}$ is obtained using the selected bandwidth $\mathbf{d}^{(j)}$.

- Step 3(ii) The estimator $\hat{\boldsymbol{\beta}}_{n}^{\dagger}$ can be approximated by $\hat{\boldsymbol{\beta}}_{n, J^{*}}^{\dagger}=$ $\sum_{j=1}^{J^{*}}\left(\left(\breve{\mathbb{X}}_{\mathbf{d}^{(j)}}^{\dagger^{\prime}} \breve{\mathbb{X}}_{\mathbf{d}^{(j)}}^{\dagger}\right)^{-1} \breve{\mathbb{X}}_{\mathbf{d}^{(j)}}^{\dagger^{\prime}} \breve{\mathbf{Y}}^{(j)}\right) / J^{*}$, and $\hat{h}_{0 n}(\cdot)$ is approximated by $\hat{h}_{0 n, J^{*}}\left(\mathbf{X}^{\ddagger}\right)=\frac{1}{J^{*}} \sum_{j=1}^{J^{*}} \frac{\sum_{i=1}^{n} K_{\mathbf{d}^{(j)}}\left(\mathbf{x}^{\ddagger}-\mathbf{X}_{i}^{\ddagger}\right)\left(\tilde{Y}_{i}^{(j)}-\mathbf{X}_{i}^{\dagger^{\prime}} \hat{\boldsymbol{\beta}}_{n, J^{*}}^{\dagger}\right)}{\sum_{i=1}^{n} K_{\mathbf{d}^{(j)}}\left(\mathbf{x}^{\ddagger}-\mathbf{X}_{i}^{\ddagger}\right)}$. 
When $J^{*}$ is sufficiently large, the estimators $\hat{\boldsymbol{\beta}}_{n, J^{*}}^{\dagger}$ and $\hat{h}_{0 n, J^{*}}$ are close approximations to $\hat{\boldsymbol{\beta}}_{n}^{\dagger}$ and $\hat{h}_{0 n}(\cdot)$.

Alternatively, we can estimate the nonparametric function $h_{0}(\cdot)$ using splines. For example, to estimate $h_{0}\left(x^{\ddagger}\right)$ by a natural cubic spline with $M$ knots, we may use $h_{0}\left(x^{\ddagger}\right)=\sum_{j=1}^{M} \alpha_{j} b_{j}\left(x^{\ddagger}\right)$. The associated spline coefficients $\alpha_{1}, \cdots, \alpha_{M}$ can then be estimated together with $\boldsymbol{\beta}^{\dagger}$ in the regression function by LSE. This yields an estimator of $F(t \mid \mathbf{x})$ if we substitute the obtained estimators $\hat{\boldsymbol{\theta}}_{n}$ and $\hat{h}(\cdot)$ into $\tilde{F}_{n}(t \mid \mathbf{x} ; \boldsymbol{\theta}, h(\cdot))$. The estimator involves the double integral presented in (3.6). We can compute this numerically via $\sum_{j=1}^{J^{*}} G\left(e^{\zeta+h\left(\mathbf{X}_{i}\right)}-L_{i}^{*} \mid B_{i}, \mathbf{X}_{i}, \delta_{1 i}^{(j)}, \delta_{2 i}^{(j)} ; \nu, \sigma, \gamma\right) / J^{*}$. Here, $\left(\delta_{1 i}^{(j)}, \delta_{2 i}^{(j)}\right)$ are obtained from Step 1 of Algorithms B1 and B2 for $i=1, \cdots, n$ and $j=$ $1, \ldots, J^{*}$

\subsubsection{Asymptotic Properties}

This section studies the asymptotic properties of the proposed estimators and presents the variance estimation. The derivation of the asymptotic properties is outlined in Section 1 of the Supplementary Material.

We use $\boldsymbol{\theta}_{0}$ and $\boldsymbol{\beta}_{0}$ to represent the true values of the parameters $\boldsymbol{\theta}$ and $\boldsymbol{\beta}$ under the model LRM-Indpt (2.4a). The proposed estimators $\hat{\boldsymbol{\theta}}_{\boldsymbol{n}}, \hat{\boldsymbol{\beta}}_{\boldsymbol{n}}$ and the estimator for the conditional probability using these estimators, $\hat{F}_{n}(t \mid \mathbf{x})$, have the following asymptotic properties.

Theorem 1 Under assumptions (A1)-(A2) and conditions $(C 1)-(C 7)$ in the $A p$ pendix, $\hat{\boldsymbol{\theta}}_{n}, \hat{\boldsymbol{\beta}}_{n}$ have the following properties:

(i) Strong Consistency: $\left\|\hat{\boldsymbol{\theta}}_{n}-\boldsymbol{\theta}_{0}\right\| \rightarrow 0$, $\left\|\hat{\boldsymbol{\beta}}_{n}-\boldsymbol{\beta}_{0}\right\| \rightarrow 0$ almost surely as $n \rightarrow \infty$.

(ii)Asymptotic Normality: $\sqrt{n}\left(\hat{\boldsymbol{\theta}}_{n}-\boldsymbol{\theta}_{0}\right) \stackrel{d}{\rightarrow} N\left(0, A V\left(\boldsymbol{\theta}_{\mathbf{0}}\right)\right)$ and $\sqrt{n}\left(\hat{\boldsymbol{\beta}}_{n}-\boldsymbol{\beta}_{0}\right) \stackrel{d}{\rightarrow}$ 
$N\left(0, A V\left(\boldsymbol{\beta}_{\mathbf{0}}\right)\right)$, where the asymptotic variances are given by

$$
\begin{aligned}
& A V\left(\boldsymbol{\theta}_{0}\right)=\Pi^{-1}\left(\boldsymbol{\theta}_{0}\right) \Sigma\left(\boldsymbol{\theta}_{0}\right) \Pi^{-1}\left(\boldsymbol{\theta}_{0}\right), \text { and } \\
& A V\left(\boldsymbol{\beta}_{0}\right)=\mathrm{E}^{-1}\left[\mathrm{X}_{i} \mathrm{X}_{i}^{\prime}\right] \operatorname{Var}\left(\mathrm{X}_{i}\left(\mathrm{E}\left[\log L_{i} \mid \mathcal{O}_{i} ; \boldsymbol{\theta}_{\mathbf{0}}\right]-\mathrm{X}_{i}^{\prime} \boldsymbol{\beta}_{0}\right)\right) E^{-1}\left[\mathrm{X}_{i} \mathrm{X}_{i}^{\prime}\right],
\end{aligned}
$$

with $\Pi(\boldsymbol{\theta})=\mathrm{E}\left[-\partial l_{o_{i}}(\boldsymbol{\theta}) / \partial \boldsymbol{\theta}\right], \Sigma(\boldsymbol{\theta})=\operatorname{Var}\left(\partial l_{o_{i}}(\boldsymbol{\theta}) / \partial \boldsymbol{\theta}\right)$.

Note that the robust sandwich variance estimator of $\hat{\boldsymbol{\theta}}_{n}$ is $V\left(\hat{\boldsymbol{\theta}}_{n}\right)$, where

$$
V(\boldsymbol{\theta})=\left(-\frac{\partial^{2} l_{n}(\boldsymbol{\theta})}{\partial \boldsymbol{\theta}^{2}}\right)^{-1}\left(\left[\frac{\partial l_{n}(\boldsymbol{\theta})}{\partial \boldsymbol{\theta}}\right]\left[\frac{\partial l_{n}(\boldsymbol{\theta})}{\partial \boldsymbol{\theta}}\right]^{\prime}\right)\left(-\frac{\partial^{2} l_{n}(\boldsymbol{\theta})}{\partial \boldsymbol{\theta}^{2}}\right)^{-1}
$$

The variance estimator of $\hat{\boldsymbol{\beta}}_{n}$ is

$$
V\left(\hat{\boldsymbol{\beta}}_{n}\right)=\left(\sum_{i=1}^{n} \mathrm{X}_{i} \mathrm{X}_{i}^{\prime}\right)^{-1} \operatorname{Var}\left(\sum_{i=1}^{n} \mathrm{X}_{i}\left(\mathrm{E}\left[\log L_{i} \mid \mathcal{O}_{i} ; \hat{\boldsymbol{\theta}}_{\boldsymbol{n}}\right]-\mathrm{X}_{i}^{\prime} \hat{\boldsymbol{\beta}}_{n}\right)\right)\left(\sum_{i=1}^{n} \mathrm{X}_{i} \mathrm{X}_{i}^{\prime}\right)^{-1}
$$

As presented in Algorithm B1, we impute possible values for the duration by generating the reporting delay $S_{i}$ for $i=1, \cdots, n$ from the IG distribution using the estimated parameters from Algorithm A and generating $\delta_{1 i}$ and $\delta_{2 i}$ from the conditional distribution. Each imputed dataset yields a least-squares estimator for $\boldsymbol{\beta}$. Following Goetghebeur and Ryan (2000), we can estimate the variance of $\hat{\boldsymbol{\beta}}_{n}$ via a weighted sum of the empirical variance of the imputation estimates and the mean of the imputation variances. The weights are $1+1 / J^{*}$ and 1 , respectively, where $J^{*}$ is the number of imputations used in ALGORITHM B. The estimated variance of $\hat{\boldsymbol{\beta}}_{n}$ is

$$
\left(1+1 / J^{*}\right) \frac{1}{J^{*}-1} \sum_{j=1}^{J^{*}}\left(\hat{\boldsymbol{\beta}}^{(j)}-\hat{\boldsymbol{\beta}}_{n}\right)^{2}+\frac{1}{J^{*}} \sum_{j=1}^{J^{*}} \hat{\operatorname{Var}}\left(\hat{\boldsymbol{\beta}}^{(j)}\right),
$$

where $\hat{\boldsymbol{\beta}}^{(j)}=\left(\mathbb{X}^{\prime} \mathbb{X}\right)^{-1} \mathbb{X}^{\prime} \tilde{\mathbf{Y}}^{(j)}$ is the least-squares estimator and $\hat{\operatorname{Var}}\left(\hat{\boldsymbol{\beta}}^{(j)}\right)$ is the estimated variance of $\hat{\boldsymbol{\beta}}^{(j)}$ with the $j^{\text {th }}$ imputed dataset. In our algorithm, we set 
$J^{*}=200$.

Further, the proposed estimator $\hat{F}_{n}(t \mid \mathbf{x})=\tilde{F}_{\epsilon, n}\left(\log t-\mathbf{x}^{\prime} \boldsymbol{\beta} ; \hat{\boldsymbol{\theta}}_{\boldsymbol{n}}, \hat{\boldsymbol{\beta}}_{\boldsymbol{n}}\right)$ has the following asymptotic property.

Theorem 2 Under assumptions (A1)-(A2) and conditions (C1)-(C7) for the loglikelihood function in (3.8), $\hat{F}_{n}(t \mid \mathbf{x})$ has the following properties with fixed $\mathbf{x}$ :

(i) Strong Consistency: $\sup _{t \in[0, \tau]}\left|\hat{F}_{n}(t \mid \mathbf{x})-F(t \mid \mathbf{x})\right| \stackrel{p}{\rightarrow} 0$ as $n \rightarrow \infty$.

(ii) Weak Convergence: For $t \in[0, \tau]$, as $n \rightarrow \infty, \sqrt{n}\left(\hat{F}_{n}(t \mid \mathbf{x})-F(t \mid \mathbf{x})\right)$ converges weakly in $\ell^{\infty}([0, \tau])$ to a tight, mean-zero Gaussian process $\mathcal{G}$ with covariance $\operatorname{Cov}(\mathcal{G}(t \mid \mathbf{x}), \mathcal{G}(s \mid \mathbf{x}))$ given by

$$
\begin{aligned}
& \int \mathrm{E}\left\{M\left(t, L_{i}^{*}, B_{i}, \mathbf{X}_{i} ; \mathbf{x}, \boldsymbol{\theta}_{\mathbf{0}}, \boldsymbol{\beta}_{\mathbf{0}}\right) M\left(s, L_{i}^{*}, B_{i}, \mathbf{X}_{i} ; \mathbf{x}, \boldsymbol{\theta}_{\mathbf{0}}, \boldsymbol{\beta}_{\mathbf{0}}\right)\right\}-F(t \mid \mathbf{x}) F(s \mid \mathbf{x}) \quad t \neq s, \\
& \mathrm{E}\left\{M\left(t, L_{i}^{*}, B_{i}, \mathbf{X}_{i} ; \mathbf{x}, \boldsymbol{\theta}_{\mathbf{0}}, \boldsymbol{\beta}_{\mathbf{0}}\right)^{2}\right\}-F^{2}(t \mid \mathbf{x}) \\
& +\mathrm{E}_{\boldsymbol{\theta}_{\mathbf{0}}, \boldsymbol{\beta}_{\mathbf{0}}}\left[\frac{\partial M\left(t, L_{i}^{*}, B_{i}, \mathbf{X}_{i} ; \mathbf{x}, \boldsymbol{\theta}, \boldsymbol{\beta}\right)}{\partial \boldsymbol{\theta}}\right]^{\prime} A V\left(\boldsymbol{\theta}_{0}\right) \mathrm{E}_{\boldsymbol{\theta}_{\mathbf{0}}, \boldsymbol{\beta}_{\mathbf{0}}}\left[\frac{\partial M\left(t, L_{i}^{*}, B_{i}, \mathbf{X}_{i} ; \mathbf{x}, \boldsymbol{\theta}, \boldsymbol{\beta}\right)}{\partial \boldsymbol{\theta}}\right] \\
& +\mathrm{E}_{\boldsymbol{\theta}_{\mathbf{0}}, \boldsymbol{\beta}_{\mathbf{0}}}\left[\frac{\partial M\left(t, L_{i}^{*}, B_{i}, \mathbf{X}_{i} ; \mathbf{x}, \boldsymbol{\theta}, \boldsymbol{\beta}\right)}{\partial \boldsymbol{\beta}}\right]^{\prime} A V\left(\boldsymbol{\beta}_{0}\right) \mathrm{E}_{\boldsymbol{\theta}_{0}, \boldsymbol{\beta}_{\mathbf{0}}}\left[\frac{\partial M\left(t, L_{i}^{*}, B_{i}, \mathbf{X}_{i} ; \mathbf{x}, \boldsymbol{\theta}, \boldsymbol{\beta}\right)}{\partial \boldsymbol{\beta}}\right] \quad t=s,
\end{aligned}
$$

where $M\left(t, L_{i}^{*}, B_{i}, \mathbf{X}_{i} ; \mathbf{x}, \boldsymbol{\theta}, \boldsymbol{\beta}\right)$ is

$$
\iint G\left(t e^{-\mathbf{x}^{\prime} \boldsymbol{\beta}_{1}+\mathbf{X}_{i}^{\prime} \boldsymbol{\beta}_{1}}-L_{i}^{*} \mid B_{i}, \mathbf{X}_{i}, \delta_{1}, \delta_{2} ; \nu, \sigma, \gamma\right) \phi_{1}\left(\delta_{1} \mid \mathcal{O}_{i} ; \boldsymbol{\theta}\right) \phi_{2}\left(\delta_{2} \mid \mathcal{O}_{i} ; \boldsymbol{\theta}\right) \mathrm{d} \delta_{1} \mathrm{~d} \delta_{2} .
$$

Note that $\mathrm{E}\left\{M\left(t, L_{i}^{*}, B_{i}, \mathbf{X}_{i} ; \mathbf{x}, \boldsymbol{\theta}_{\mathbf{0}}, \boldsymbol{\beta}_{\mathbf{0}}\right)^{2}\right\}$ can be approximated by the average $\sum_{i=1}^{n}\left[\sum_{j=1}^{J^{*}} G\left(t e^{-\mathbf{x} \boldsymbol{\beta}+\mathbf{X}_{i} \boldsymbol{\beta}}-L_{i}^{*} \mid B_{i}, \mathbf{X}_{i}, \delta_{1 i}^{(j)}, \delta_{2 i}^{(j)} ; \hat{\nu}_{n}, \hat{\sigma}_{n}, \hat{\boldsymbol{\gamma}}_{n}\right) / J^{*}\right]^{2} / n$ with $\left(\delta_{1 i}^{(j)}, \delta_{2 i}^{(j)}\right)$ obtained from Step 1 of Algorithm B1 for $j=1, \cdots, J^{*}$. This strategy can be used to approximate $\mathrm{E}_{\boldsymbol{\theta}_{\mathbf{0}}, \boldsymbol{\beta}_{\mathbf{0}}}\left[\partial M\left(t, L_{i}^{*}, B_{i}, \mathbf{X}_{i} ; \mathbf{x}, \boldsymbol{\theta}, \boldsymbol{\beta}\right) / \partial \boldsymbol{\theta}\right]$ and $\mathrm{E}_{\boldsymbol{\theta}_{\mathbf{0}}, \boldsymbol{\beta}_{\mathbf{0}}}\left[\partial M\left(t, L_{i}^{*}, B_{i}, \mathbf{X}_{i} ; \mathbf{x}, \boldsymbol{\theta}, \boldsymbol{\beta}\right) / \partial \boldsymbol{\beta}\right]$.

The proposed estimator of $\boldsymbol{\beta}^{\dagger}$ in the PLRM-Indpt 2.4b) with kernel smoothing also has the asymptotic properties.

Theorem 3 Under assumptions (A1)-(A2) and conditions (C8)-(C10) in the Appendix, $\hat{\boldsymbol{\beta}}_{n}^{\dagger}$ has the following properties:

(i) Strong Consistency: $\left\|\hat{\boldsymbol{\beta}}_{n}^{\dagger}-\boldsymbol{\beta}_{0}^{\dagger}\right\| \rightarrow 0$ almost surely as $n \rightarrow \infty$. 
(ii) Asymptotic Normality: $\sqrt{n}\left(\hat{\boldsymbol{\beta}}_{n}^{\dagger}-\boldsymbol{\beta}_{0}^{\dagger}\right) \stackrel{d}{\rightarrow} N\left(0, A V\left(\boldsymbol{\beta}_{\mathbf{0}}^{\dagger}\right)\right)$, where the asymptotic variances are given by

$$
A V\left(\boldsymbol{\beta}_{0}^{\dagger}\right)=\sigma_{\epsilon}^{2} \boldsymbol{\Omega}^{-1} \boldsymbol{\Xi} \boldsymbol{\Omega}^{-1},
$$

with $\boldsymbol{\Omega}=\left(\breve{\mathbb{X}}^{\dagger^{\prime}} \breve{\mathbb{X}}^{\dagger}\right)^{-1}, \boldsymbol{\Xi}=\breve{\mathbb{X}}^{\dagger^{\prime}}\left(\mathbf{I}-\mathbf{H}_{\mathbf{d}}\right)\left(\mathbf{I}-\mathbf{H}_{\mathbf{d}}\right)^{\prime \mathbb{X}^{\dagger}}$, and $\sigma_{\epsilon}^{2}=\operatorname{Var}\left(\mathrm{E}\left[\log L_{i} \mid \mathcal{O}_{i}\right]-\right.$ $\left.\mathbf{X}_{i}^{\dagger} \boldsymbol{\beta}^{\dagger}-h_{0}\left(\mathbf{X}_{i}^{\ddagger}\right)\right)$.

With Algorithm B2, we can also estimate the variance of $\hat{\boldsymbol{\beta}}_{n}^{\dagger}$ using equation 3.12. Here, $\hat{\boldsymbol{\beta}}^{(j)}=\left(\breve{\mathbb{X}}_{\mathbf{d}^{(j)}}^{\dagger^{\prime}} \breve{\mathbb{X}}_{\mathbf{d}^{(j)}}^{\dagger}\right)^{-1} \breve{\mathbb{X}}_{\left.\mathbf{d}^{\prime}{ }^{\prime}\right)} \breve{\mathbf{Y}}^{(j)}, \hat{\operatorname{Var}}\left(\hat{\boldsymbol{\beta}}^{(j)}\right)=\widetilde{\sigma^{(j)}{ }_{\epsilon}{ }^{2}} \boldsymbol{\Omega}^{-1} \boldsymbol{\Xi} \boldsymbol{\Omega}^{-1}$, and $\widetilde{\sigma^{(j)}{ }_{\epsilon}^{2}}=\operatorname{Var}\left(\tilde{Y}_{i}^{(j)}-\mathbf{X}_{i}^{\dagger} \hat{\boldsymbol{\beta}}^{(j)}-h_{0}\left(\mathbf{X}_{i}^{\ddagger}\right)\right)$.

\subsection{Estimation with Spatially Correlated Study Units}

The estimation procedures (Section 3.1) for both the conditional distribution and the regression parameters can be extended to accommodate spatial correlation in the data. In addition to the assumption (A2) given in Section 3.1, we make the following assumption throughout this section:

Assumption $\left(\mathrm{A} 1^{*}\right)$. Let $\mathcal{X}=\left(\mathbf{X}_{1}, \cdots, \mathbf{X}_{n}\right)^{\prime}$. Conditional on $\left(\boldsymbol{\delta}_{1}, \boldsymbol{\delta}_{2}, \boldsymbol{\mathcal { X }}\right)$, assume that $\left(T_{R i}, T_{F i}, B_{i}, D_{i}\right)$ is independent of $\left(T_{R j}, T_{F j}, B_{j}, D_{j}\right)$ for $i, j=$ $1, \cdots, n$ and $i \neq j$.

The assumption $\left(A 1^{*}\right)$ implies that realizations are independent conditional on the covariates and the random effects. This is plausible because we use correlated random effects to accommodate the spatial correlation. From Model (2.4), the conditional distribution function $F(t \mid \mathbf{x})=P\left(L_{i} \leq t \mid \mathbf{X}_{i}\right)$ can be written as $\iint F_{\epsilon}\left(\log t-\left(h\left(\mathbf{x}, \delta_{1}, \delta_{2}\right)\right)\right) \phi_{1}\left(\delta_{1} ; \psi_{1}\right) \phi_{2}\left(\delta_{2} ; \psi_{2}\right) d \delta_{1} d \delta_{2}$. Here $\phi_{1}\left(\cdot ; \psi_{1}\right)$ and $\phi_{2}\left(\cdot ; \psi_{2}\right)$ are density functions of the marginal distributions of $\delta_{1 i}$ and $\delta_{2 i}$ for $i=1, \cdots, n$, i.e. the density functions for $N\left(0, \psi_{1}^{2}\right)$ and $N\left(0, \psi_{2}^{2}\right)$.

We employ the techniques of Section 3.1.1 and estimate $F_{\epsilon}(\zeta)$ by $\tilde{F}_{\epsilon, n}(\zeta ; \boldsymbol{\theta}, h(\cdot))=$ 
$n^{-1} \sum_{i=1}^{n} P\left(S_{i} \leq e^{\zeta+h\left(\mathbf{X}_{i}, \delta_{1}, \delta_{2}\right)}-L_{i}^{*} \mid \mathcal{O}_{i}\right)$ with $P\left(S_{i} \leq e^{\zeta+h\left(\mathbf{X}_{i}, \delta_{1}, \delta_{2}\right)}-L_{i}^{*} \mid \mathcal{O}_{i}\right)$ being $\iint G\left(e^{\zeta+h\left(\mathbf{X}_{i}, \delta_{1}, \delta_{2}\right)}-L_{i}^{*} \mid B_{i}, \mathbf{X}_{i}, \delta_{1}, \delta_{2} ; \nu, \sigma, \gamma\right) \phi_{1}\left(\delta_{1} \mid \mathcal{O}_{i} ; \boldsymbol{\theta}\right) \phi_{2}\left(\delta_{2} \mid \mathcal{O}_{i} ; \boldsymbol{\theta}\right) \mathrm{d} \delta_{1} \mathrm{~d} \delta_{2}$ with $\boldsymbol{\theta}=$ $\left(\nu, \sigma, \gamma, \psi_{1}, \rho_{1}, \psi_{2}, \rho_{2}\right)$ and $h(\cdot)$ being specified in LRM-Corrltd (2.4c) and PLRMCorrltd (2.4d). This estimator $\tilde{F}_{\epsilon, n}(\cdot ; \boldsymbol{\theta}, h(\cdot))$ can then be used to estimate the conditional distribution. Denote the resulting estimator by $\tilde{F}_{n}(t \mid \mathbf{x} ; \boldsymbol{\theta}, h(\cdot))$. With the estimators of $\boldsymbol{\theta}$ and $h(\cdot)$, the applicable estimator, denoted $\hat{F}_{n}(t \mid \mathbf{x})$, can be obtained $\operatorname{via} \hat{F}_{n}(t \mid \mathbf{x})=\tilde{F}_{n}(t \mid \mathbf{x} ; \hat{\boldsymbol{\theta}}, \hat{h}(\cdot))$.

Under assumptions $\left(\mathrm{A}^{*}\right)$ and $(\mathrm{A} 2)$, the log-likelihood function with observed data conditional on $\mathcal{X}$, is $\log L_{o b s \mid \mathcal{X}}(\boldsymbol{\theta})=\log \iint\left\{L_{o b s \mid \boldsymbol{\delta}_{\mathbf{1}}, \boldsymbol{\delta}_{\mathbf{2}}, \mathcal{X}}(\nu, \sigma, \boldsymbol{\gamma})\right\} d\left[\boldsymbol{\delta}_{1}, \boldsymbol{\delta}_{2}\right]$, with $L_{o b s \mid \boldsymbol{\delta}_{\mathbf{1}}, \boldsymbol{\delta}_{\mathbf{2}}, \mathcal{X}}=\prod_{i=1}^{n} \int_{0}^{\infty}\left\{L_{o b s \mid S, \delta_{1 i}, \delta_{2 i}, \mathbf{X}_{i}}\right\} d\left[S_{i} \mid \delta_{1 i}, \delta_{2 i}, \mathbf{X}_{i}\right]$ and $L_{o b s \mid S, \delta_{1 i}, \delta_{2 i}, \mathbf{X}_{i}}$ being the product of the two densities $\left[D_{i} \mid B_{i}, L_{i}^{*}, \delta_{1 i}, \delta_{2 i}, \mathbf{X}_{i}\right]$ and $\left[B_{i} \mid S_{i}, \delta_{1 i}, \delta_{2 i}, \mathbf{X}_{i}\right]$. The MCEM algorithm can be employed to compute the MLE of $\boldsymbol{\theta}$.

We now consider the full dataset, which is the observed data 2.1 augmented by $\underline{S}=\left\{S_{i}, i=1,2, \cdots, n\right\}, \underline{\delta_{1}}=\left\{\delta_{1 i}, i=1,2, \cdots, n\right\}$, and $\underline{\delta_{2}}=\left\{\delta_{2 i}, i=\right.$ $1,2, \cdots, n\}$. The full-data $\log$-conditional likelihood function is $l_{F}^{*}\left(\boldsymbol{\theta} \mid \mathcal{O}, \underline{S}, \underline{\delta}_{1}, \underline{\delta}_{2}\right)=$ $l_{F_{1}}^{*}\left(\nu, \sigma, \gamma \mid \underline{S}, \underline{\delta}_{1}, \underline{\delta}_{2}\right)+l_{F_{2}}^{*}\left(\boldsymbol{\theta} ; \underline{S}, \underline{\delta}_{1}, \underline{\delta}_{2}\right)$, where $l_{F_{1}}^{*}$ is the same as $l_{F_{1}}$ under the independence assumption and $l_{F_{2}}^{*}(\boldsymbol{\theta} ; \underline{S}, \underline{\delta})=\sum_{i=1}^{n} \log \left[S_{i} \mid \delta_{1 i}, \delta_{2 i}, \mathbf{X}_{i}\right]-1 / 2 \log \left|\Sigma\left(\psi_{1}, \rho_{1}\right)\right|-$ $1 / 2 \underline{\delta}_{1}{ }^{\prime} \Sigma^{-1}\left(\psi_{1}, \rho_{1}\right) \underline{\delta}_{1}-1 / 2 \log \left|\Sigma\left(\psi_{2}, \rho_{2}\right)\right|-1 / 2 \underline{\delta}_{2}{ }^{\prime} \Sigma^{-1}\left(\psi_{2}, \rho_{2}\right) \underline{\delta}_{2}$. The algorithm is similar to Algorithm A in Section 3.1.2; the main difference is the generation of $\underline{\delta}_{1}^{(j)}, \underline{\delta}_{2}^{(j)}$ for the E-step. We generate the random vector $\left(\delta_{11}^{(j)}, \cdots, \delta_{1 n}^{(j)}, \delta_{21}^{(j)}, \cdots, \delta_{2 n}^{(j)}\right)$ for $j=1, \cdots, J$ from $\boldsymbol{\phi}^{(m+1)}\left(\boldsymbol{\delta}_{1}, \boldsymbol{\delta}_{2} \mid \mathcal{O} ; \boldsymbol{\theta}^{(m)}\right)$, which is the conditional distribution of $\boldsymbol{\delta}_{1}, \boldsymbol{\delta}_{2}$ given the observed data with $\boldsymbol{\theta}^{(m)}$ and can be expressed as

$$
\frac{L_{o b s \mid \boldsymbol{\delta}_{\mathbf{1}}, \boldsymbol{\delta}_{\mathbf{2}}, \mathcal{X}}\left(\nu^{(m)}, \sigma^{(m)}, \boldsymbol{\gamma}^{(m)} ; \boldsymbol{\delta}_{\mathbf{1}}, \boldsymbol{\delta}_{\mathbf{2}}\right) \boldsymbol{\phi}_{1}\left(\boldsymbol{\delta}_{\mathbf{1}} ; \psi_{1}^{(m)}, \rho_{1}^{(m)}\right) \boldsymbol{\phi}_{2}\left(\boldsymbol{\delta}_{\mathbf{2}} ; \psi_{2}^{(m)}, \rho_{2}^{(m)}\right)}{\iint L_{o b s \mid \boldsymbol{\delta}_{\mathbf{1}}, \boldsymbol{\delta}_{\mathbf{2}}, \mathcal{X}}\left(\nu^{(m)}, \sigma^{(m)}, \boldsymbol{\gamma}^{(m)} ; \boldsymbol{\delta}_{\mathbf{1}}, \boldsymbol{\delta}_{\mathbf{2}}\right) \boldsymbol{\phi}_{1}\left(\boldsymbol{\delta}_{\mathbf{1}} ; \psi_{1}^{(m)}, \rho_{1}^{(m)}\right) \boldsymbol{\phi}_{2}\left(\boldsymbol{\delta}_{\mathbf{2}} ; \psi_{2}^{(m)}, \rho_{2}^{(m)}\right) \mathrm{d} \boldsymbol{\delta}_{\mathbf{1}} d \boldsymbol{\delta}_{\mathbf{2}}},
$$


where $\boldsymbol{\phi}_{1}\left(\cdot, \psi_{1}, \rho_{1}\right), \boldsymbol{\phi}_{2}\left(\cdot, \psi_{2}, \rho_{2}\right)$ are the density functions of multivariate normal distributions with the covariance functions specified in 2.3 . The Metropolis-Hastings algorithm can be used to generate $\left(\delta_{11}^{(j)}, \cdots, \delta_{1 n}^{(j)}, \delta_{21}^{(j)}, \cdots, \delta_{2 n}^{(j)}\right)$.

We use the regression model (2.4) to adjust for spatial correlation. AlgorithmS B1 and B2 can be applied to estimate the regression function $h(\cdot)$ with Models LRM-Corrltd (2.4c) and PLRM-Corrltd (2.4d). The shared random effects can be predicted using the conditional distribution $\boldsymbol{\phi}\left(\boldsymbol{\delta}_{1}, \boldsymbol{\delta}_{2} \mid \mathcal{O} ; \hat{\boldsymbol{\theta}}\right)$ with $\hat{\boldsymbol{\theta}}$ obtained by the MCEM algorithm.

The robust sandwich variance estimator given in Theorem 1 can be used to estimate the variances of $\hat{\boldsymbol{\theta}}_{n}$. For the variance estimation of the regression parameters in Models LRM-Corrltd (2.4c) and PLRM-Corrltd (2.4d), we can apply the imputation methods described in Section 3.1.3.

\section{Analysis of Alberta Wildfire Data}

In this section, we conduct regression analysis of Alberta wildfire data to illustrate our approach. The duration of interest is the ISA duration. The goal is to study the association of the ISA duration with a list of risk factors and then to consider prediction based on this. The time of the initial attack is when the first fire-fighting resource arrives to prevent further extension of the wildfire. It is believed that fires with a longer ISA duration may require more effort to suppress, so this interval is valuable information for fire management agencies. Xiong, Braun, and $\mathrm{Hu}(2021)$ estimated the distribution of the ISA duration separately for fires from the upper and lower regions of Alberta, finding that the distribution depends on the region. To quantify the association of the ISA duration with the risk factors, we conduct a regression analysis. 
The dataset includes 603 lightning-caused fires in Alberta in 2004 during the fire season, May to August. There are six risk factors, region (upper or lower) and fuel type (C1, C2, M2, Others), and three weather variables, temperature, relative humidity, and wind speed. All of the weather variables are recorded on the reported time of fires. See Tables S1 and S2 in the Supplementary Materials for the summary statistics. The upper region covers Fort McMurray, High Level, Lac La Biche, Peace River, and Slave Lake; the lower region covers Calgary, Edson, Grande Prairie, Rocky Mountain House, and Whitecourt. The three most common fuel types are C1 (Spruce-lichen woodland), C2 (Boreal spruce), and M2 (Boreal mixedwoodGreen). The remaining fuel types are categorized into Other Types. For detailed characteristics and photographs of these fuel types, see Forestry Canada Fire Danger Group (1992). Fires that were large at the report time occurred more frequently in the upper region, and there were multiple large fires close to each other (see Figure S1 in the Supplementary Materials for a map of lightning-caused fires in the dataset).

There appears to be a strong correlation between each pair of weather variables. In addition, temperature and relative humidity are closely associated with region; see the pairwise correlation plot in Figure $\mathrm{S} 2$ of the Supplementary Material for example. Based on these findings, we select potential covariates via forward selection using the Akaike information criterion (AIC). The potential covariates considered are region, fuel type, wind speed. The baseline category for Region is the lower region, and the baseline for Fuel type is other types.

We consider two cases of random drift $\nu_{i}$ in the longitudinal model (2.2): (1) with a random intercept $\nu_{i}=\nu e^{\mathbf{X}^{\prime}{ }_{i} \boldsymbol{\gamma}+\delta_{1 i}}$ for $i=1, \cdots, n$; (2) with the mixed effect $\nu_{i}=\nu e^{\mathbf{X}^{\prime}{ }_{i} \boldsymbol{\gamma}+X^{*}{ }_{i} \delta_{2 i}+\delta_{1 i}}$, where $X^{*}$ denotes the factor Region. We estimate $\boldsymbol{\theta}$ and all 
the parameters involved in Model (2.2), assuming first that study units are independent and then that they are spatially correlated. Table 1 presents the estimates for $\boldsymbol{\theta}$. The standard errors are estimated using the sandwich variance estimator, and the significant effects are presented in boldface. For the mixed effects, the estimates of $\psi_{1}, \psi_{2}$ indicate considerable variation among fires; the variation also depends on the region. The estimated standard errors of the parameters for the spatially correlated case are smaller than those for the independent case, although the parameter estimates themselves are close.

The estimates of $\gamma$ can be used to describe the effect of the risk factors on the burnt area process. The analysis assuming spatial correlation indicates that fires in the upper region or reported on days with a higher wind speed tend to have a larger drift and may grow faster. This analysis also indicates that fires with M2 fuels tend to have a smaller drift than fires with $\mathrm{C} 1, \mathrm{C} 2$, or Others. This finding agrees with analyses of the differences in burn rate among the fuel types of Alberta Cumming, 2001; Tremblay, Duchesne, and Cumming, 2018).

Table 1 is here.

We consider LRMs and PLRMs with assumed independent and correlated study units. For the PLRMs, the covariates in the parametric component $\mathbf{X}^{\dagger}$ are the variables Region and Fuel type, and a smooth function of Wind speed is included. We use kernel smoothing and natural cubic splines to estimate the nonparametric term. Tables 2 and 3 present the estimates of the regression parameters in LRMs and PLRMs with kernel smoothing. We also estimate the LRM parameters using two conventional approaches. The first uses $L^{*}$ for the regression analysis, and the second treats the fire data as interval-censored, i.e. $L_{i} \in\left[L_{i}^{*}, L_{i}^{*}+R_{\max }\right]$, with $R_{\max }$ being the longest reporting delay. We set $R_{\max }=12,48$ hours. In the interval- 
censored case, we use the R-package smoothsurv (Komárek and Komárek, 2015) to estimate $\boldsymbol{\beta}$. For the correlated units, we use the regression model given in PLRMCorrltd $2.4 \mathrm{~d})$ with two conventional approaches, where $\left(\delta_{1 i}, \delta_{2 i}\right)$ can be obtained as a realization from the last iteration of the MCEM algorithm in Section 3.1.2. The results for both the LRM and PLRM indicate that region is a significant predictor for the ISA duration, whereas the two conventional approaches do not reveal this significant association. The positive estimate of $\beta_{\text {region }}$ suggests that fires occurring in the upper region tend to have a longer ISA duration, which is contrary to the estimates based on the observed ISA duration. The wildfire areas in the lower region are closer to Edmonton and Calgary, which are Alberta's two metropolitan areas with populations exceeding one million. There is more infrastructure for getting fire crews and other resources to suppress fires in this region quickly. Hence, the ISA durations are expected to be shorter. On the other hand, fires in the upper region are harder to get to and are not detected as readily, leading to longer ISA durations. Similar conclusions can be drawn from Table S3 in the Supplementary Material for the estimates of PLRMs with natural cubic splines.

Under the LRM, the ISA duration is not significantly associated with the explanatory variables wind speed and fuel type. This may be caused by the correlation between these two independent variables; our simulation confirms this finding. On the other hand, the estimate $\beta_{M 2}$ is significantly different from 0 with PLRMs with spatial correlation. This indicates that the ISA duration is shorter for fires with M2 fuel than for fires with other fuel types. We plot the estimated nonparametric function $h_{0}(\cdot)$ of Wind speed in Figure 3 for Models PLRM-Indpt 2.4b) and PLRMCorrltd (2.4d). For comparison, a reference line at $y=0$ is overlaid, together with pointwise $95 \%$ confidence intervals of $\hat{h}_{0}(\cdot)$, which is obtained from the $2.5 \%$ and 
97.5\% quantiles of the realizations of $\hat{h}_{0}$ using a bootstrap procedure (described below) with a resample size of 1000 .

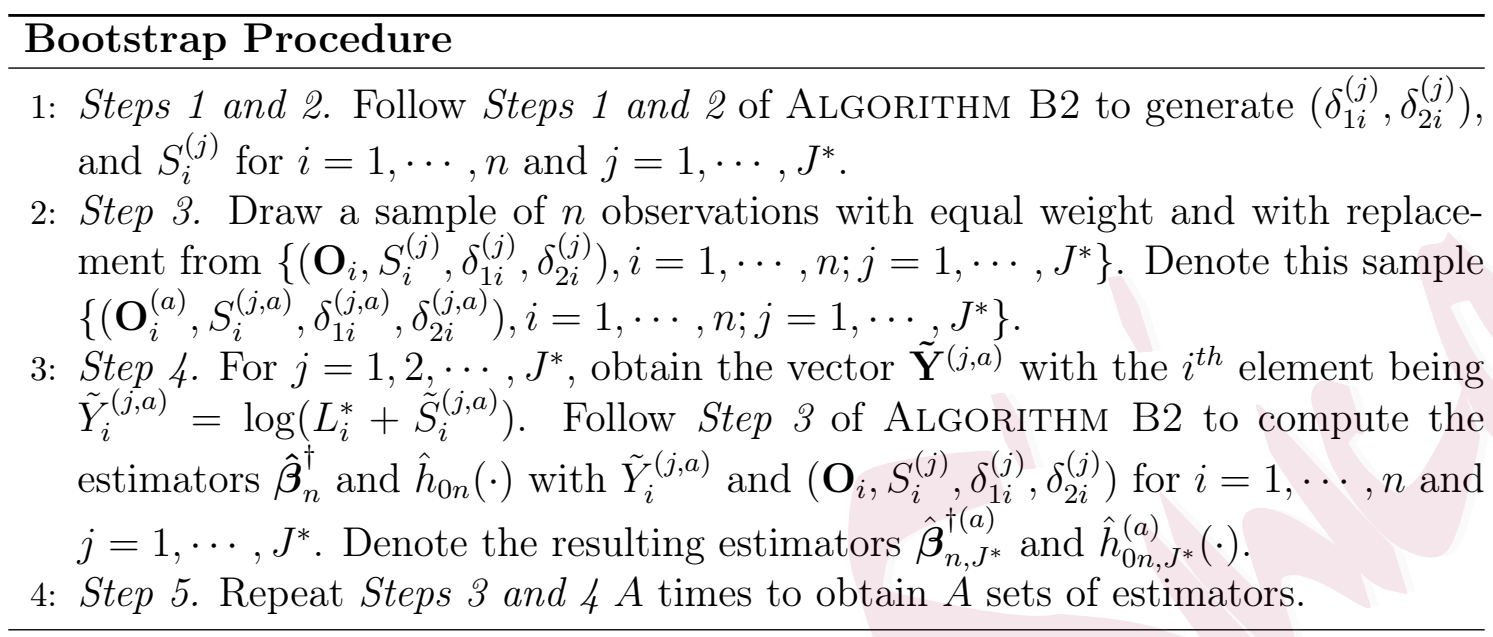

The two nonparametric estimates by kernel smoothing and natural cubic splines under the two models are clearly different from zero. This indicates that it is necessary to use an unspecified function to capture the true association of the ISA duration with the wind speed. When the wind speed is low, it is not significantly associated with the ISA duration; the association strengthens as the speed increases.

Figure 3 is here.

_- Tables 2 and 1 are here.

We present the distribution estimates for the models LRM-Indpt 2.4a and LRM-Corrltd (2.4c) in Figure 2. For fixed values of Wind Speed, we evaluate the estimator $\hat{F}_{n}(t \mid \mathbf{x})$ in 3.7$)$. The $95 \%$ pointwise confidence intervals (CIs) are also provided. The CIs are calculated using the estimated asymptotic variance given in (3.13) for the analysis in the independent case, and they are obtained using the bootstrap variance in the correlated case. In each plot, we overlay estimated distribution curves with two conventional LRM approaches: they use the observed portion of the ISA duration and the interval-censored ISA duration. The distribution curves estimated by these approaches are similar for different subgroups of fires. 
However, the curves of the proposed estimator show that fires from the upper region are likely to have a longer ISA duration for fixed fuel type and wind speed. Moreover, M2 fires tend to have a shorter ISA duration. This shows that caution is required when dealing with durations with missing origins.

—_ Figure 2 is here. - -

Following Xiong et al. (2019), we apply a spatio-temporal extension of Moran's I test to check the validity of Models (2.4a), 2.4b), 2.4c), and (2.4d). For each model, we compute one set of the residuals via $e_{i}^{(j)}=\log \left(L_{i}^{*}+\tilde{S}_{i}^{(j)}\right)-\hat{Y}_{i}$ for $i=1, \cdots, n$, where $\tilde{S}_{i}^{(j)}$ is the imputed reporting delay based on Step 2 of ALGORITHMS B1 and B2, and $\hat{Y}_{i}$ is the fitted value obtained by each model. To construct the Moran's $I$ test statistic, we define fire neighbours to be any two fires from the same management area and with the time lag between their report times bounded by a predetermined $\tau$. The plots of Figure 4 compare Moran's $I$, evaluated at different values of $\tau$, with the LRM and PLRM. For independent units, Figure 4a shows that for the PLRM the Moran's $I$ is covered by the $95 \%$ acceptance regions when $\tau \geq 25$. As observed by Xiong et al. (2019), this suggests that the PLRM has captured the nonlinear spatial pattern and appears to be more appropriate than the LRM. For correlated units, Figure $4 \mathrm{~b}$ shows that for both models the Moran's $I$ is within the acceptance region. This indicates that spatial correlation is the main cause of the discrepancy between the value of Moran's $I$ and the expected value under the null hypothesis.

- Figure 4 is here.

Residual plots also support the Moran's I results. The residuals from the PLRM are near zero for both independent and correlated units; see scatterplots of the residuals versus wind speed and report time in Figures S3 and S4 in the Supplementary Material. These findings suggest that Models PLRM-Indpt 2.4b and 
PLRM-Corrltd(2.4d $)$ adequately capture the association of the ISA duration with the risk factors.

\section{Simulation Studies}

We carried out simulation studies to examine the finite-sample performance of our approach and to verify the findings of the real-data analysis. Simulation A generated longitudinal measurements from the Wiener process model to verify the consistency and efficiency of our approach. Simulation B generated a situation where the covariates are related by a latent variable and Simulation $C$ assessed impact of missing covariates.

\subsection{Simulation A: Study to Verify Consistency and Efficiency}

To mimic the fire data, we simulated a dataset with $n=500$ fires as follows:

\section{Settings for Simulation A}

1: Let $\mathbf{X}_{i}=\left(X_{1 i}, X_{2 i}\right)$ and $X_{1 i} \sim \operatorname{Unif}(0,1), X_{2 i} \sim B(1,0.6)$ for $i=1, \cdots, n$.

2: Generate independently $n$ locations $\boldsymbol{\omega}_{i}$ with each of the two location indices $\omega_{1 i}$ and $\omega_{2 i}$ from $\operatorname{Unif}(0,1)$.

3: Generate the burnt area process $A_{i}(t), t \in[0,2500]$ for $i=1, \cdots, n$ based on the model $A_{i}(t)=\nu_{i} t+\sigma W_{i}(t)$, where $\nu_{i}=\nu \exp \left\{X_{1 i} \gamma_{1}+X_{2 i}\left(\gamma_{2}+\delta_{2 i}\right)+\delta_{1 i}\right\}$. Let $\boldsymbol{\delta}_{1}=\left(\delta_{11}, \cdots, \delta_{1 n}\right)^{\prime}$ and $\boldsymbol{\delta}_{2}=\left(\delta_{21}, \cdots, \delta_{2 n}\right)^{\prime}$. Generate $\boldsymbol{\delta}_{1}$ and $\boldsymbol{\delta}_{2}$ from $M V N\left(0, \Sigma_{1}\right)$ and $M V N\left(0, \Sigma_{2}\right)$, respectively. To simulate different types of study units, we considered two types of covariance matrices $\Sigma_{1}$ and $\Sigma_{2}$ :

Type 1 (independent): $\Sigma_{1}=\psi_{1}^{2} \mathbf{I}$ and $\Sigma_{2}=\psi_{2}^{2} \mathbf{I}$ with $\mathbf{I}$ the $n \times n$ identity matrix.

Type 2 (spatially correlated): The $(i, j)^{t h}$ elements of $\Sigma_{1}$ and $\Sigma_{2}$ are given by $\left(\Sigma_{1}\right)_{i j}=\psi_{1}^{2} \exp \left\{-\left\|\boldsymbol{\omega}_{\boldsymbol{i}}-\boldsymbol{\omega}_{\boldsymbol{j}}\right\| / \rho_{1}\right\}$, and $\left(\Sigma_{2}\right)_{i j}=$ $\psi_{2}^{2} \exp \left\{-\left\|\boldsymbol{\omega}_{\boldsymbol{i}}-\boldsymbol{\omega}_{\boldsymbol{j}}\right\| / \rho_{2}\right\}$.

4: Let $\mu_{B}\left(\mathbf{X}_{i}\right)=0.8+2.5 X_{1 i}-X_{2 i}$. Generate the size at the report time $B_{i} \sim$ $\log \operatorname{Normal}\left(\mu_{B}\left(\mathbf{X}_{i}\right), 0.6^{2}\right)$.

5: Determine the reporting delay as $S_{i}=\max \left\{t \mid t \in[0,2500], A_{i}(t) \leq B_{i}\right\}$.

6: Generate $L_{i}^{*} \sim \operatorname{Exp}\left(\lambda_{i}\right)$, where $\lambda_{i}=\exp \left(0.1 \delta_{1 i}+X_{1 i}-\left(0.2+\delta_{2 i}\right) X_{2 i}-0.6\right)$.

7: Obtain $L_{i}=S_{i}+L_{i}^{*}, A_{i}\left(L_{i}\right)$, and $D_{i}=A_{i}\left(L_{i}\right)-B_{i}$.

When $\psi_{2}=0$, only the random intercept term $\delta_{1 i}$ is included in $\nu_{i}$. We consider 
the following four scenarios:

(A1.1) $\psi_{1}=0.25, \psi_{2}=0$, Type 1 covariance matrix.

(A1.2) $\psi_{1}=0.25, \psi_{2}=0.15$, Type 1 covariance matrix.

(A2.1) $\psi_{1}=0.25, \psi_{2}=0, \rho_{1}=0.3, \rho_{2}=0$, Type 2 covariance matrix.

(A2.2) $\psi_{1}=0.25, \psi_{2}=0.15, \rho_{1}=0.3, \rho_{2}=0.1$, Type 2 covariance matrix.

We set $\nu=3.5, \sigma=0.8, \gamma_{1}=0.2$, and $\gamma_{2}=0.15$ for all the scenarios. For each generated dataset, we considered the models LRM-Indpt 2.4a) and LRM-Corrltd (2.4c), and evaluated the estimators for independent and correlated study units. We calculated the estimated standard errors (SEs) of the estimates of $\boldsymbol{\theta}$ using the robust sandwich variance estimator and estimated the SE of the estimates of $\boldsymbol{\beta}$ via 3.12$)$. We also evaluated the least-squares estimator of $\boldsymbol{\beta}$ using $L$ and the observed $L^{*}$, as well as the interval-censored observations with $L_{i} \in\left[L_{i}^{*}, L_{i}^{*}+R_{\max }\right]$, where $R_{\max }$ is the third quantile of $S$ for each generated dataset.

In each of the four scenarios, we repeated the simulation 400 times. The sample means of the estimates under a correctly specified $\nu_{i}$ are close to the true parameter values. For example, the true specification of $\nu_{i}$ in Scenario (A1.2) is $\nu_{i}=\nu \exp \left\{\delta_{1 i}+\right.$ $\left.X_{1 i} \gamma_{1}+X_{2 i} \gamma_{2}\right\}$. The sample means of the estimates from our approaches for both independent and correlated study units are close to the true parameter values. This provides empirical evidence for the consistency of our estimator. On the other hand, the estimate of $\boldsymbol{\beta}$ from the naïve approach using the $L_{i}^{*}$ 's has a large bias and is in the opposite direction to that based on the true ISA duration and that from our approach. This shows that approaches using only the observed or interval-censored ISA duration could be misleading. See Tables S4 S7 in the Supplementary Material for the parameter estimates based on the 400 simulation repetitions in the four scenarios.

We also examined the performance of the SE estimators. The sample means 
of the estimated SEs under the true settings agree well with the empirical SEs, i.e. the sample SEs. For correlated data, both the sample SEs and the sample means of the estimated SEs under the independence assumption are larger than the values that account for the correlation. This suggests that failing to accommodate the correlation may lead to inefficient estimators.

In Scenario (A2.2), we estimated the distribution curves by our approach with correlation and obtained the estimates by the empirical distribution function of the residuals from the regression model with the true (complete) ISA duration, the observed ISA duration, and the interval-censored ISA duration. The sample means of the conditional distribution estimates using our approach are close to the empirical distribution function estimates using the complete ISA duration. We observe that fires with $X_{2}=0$ are likely to have a longer ISA duration. In sharp contrast, estimates from the two conventional approaches show the opposite behaviour. This suggests that the reporting delay has an impact on the association of the ISA duration with the covariates and should be accounted for. We also evaluated the distribution estimator by our approach under the independence assumption in the Scenarios (A2.2) with correlated units. The estimated conditional distribution curve by our approach shows similar behaviour, but with a wider $95 \%$ confidence interval than the one with correlation. This indicates that a more efficient distribution estimator can be obtained if the correlation is taken into account. The estimated distribution curves are presented in Figures $\mathbf{5 5}$ and $\mathbf{S 6}$ of the Supplementary Material. 


\subsection{Simulation B: Study to Examine the Impact of Correlated Covari- ates}

In this study, we generated covariates $X_{1}$ and $X_{2}$ that depend on a latent variable $M$. We first simulated $M_{i} \sim B(1,0.7)$ for $i=1, \cdots, n$ and then generated $X_{i 1} \sim \operatorname{Unif}\left(0, a_{1}+M_{i} b_{1}\right), X_{i 2} \sim B\left(1, \frac{\exp \left(a_{2}+M_{i} b_{2}\right)}{1+\exp \left(a_{2}+M_{i} b_{2}\right)}\right)$ for $i=1,2, \cdots, n$. We used $\left(a_{1}, b_{1}, a_{2}, b_{2}\right)=(0.9,0.1,4,-2)$ to simulate $X_{1}$ and $X_{2}$ with a Pearson correlation coefficient of -0.65 . The $\nu_{i}$ 's included only the random intercept term, and the covariance matrix was Type 2. All the other variables were as in Simulation A. We evaluated the parameter estimators using the simulated data.

The sample means of the estimates for $\boldsymbol{\theta}$ under the correct specification of $\nu_{i}$ based on 400 repetitions with the model including both $X_{1}$ and $X_{2}$ as covariates are close to the true values, and the estimates of $\gamma_{1}$ and $\gamma_{2}$ suggest that $X_{1}$ and $X_{2}$ are both significantly associated with the longitudinal process. However, $\boldsymbol{\beta}$ appears to be underestimated. Only $X_{2}$ is identified as a significant predictor for the ISA duration. This finding corroborates the observation from the real-data analysis that the ISA duration is not significantly associated with wind speed and fuel type, while these factors are associated with the burnt area process and were therefore expected to be important for the ISA duration. The simulation outcomes are presented in Table $\mathrm{S} 8$ of the Supplementary Material. We also examined the parameter estimates for the model including only $X_{1}$ or $X_{2}$, which are shown in Tables S9 and S10 of the Supplementary Material. These results show that $X_{1}$ and $X_{2}$ are significant predictors when only one of them is included in the model. This suggests that the aforementioned under-estimation of $\boldsymbol{\beta}$ may be caused by the strong correlation of $X_{1}$ and $X_{2}$. Further investigation of the variable selection or a stratified data analysis would be desirable. 


\subsection{Simulation C: Study to Examine the Impact of Missing Covariates}

This simulation examines the impact of missing covariates under two scenarios: (C.1) a missing covariate in the longitudinal process and the regression model, and (C.2) a missing covariate in the spatial correlation function.

With Scenario (C.1), we generated the longitudinal measures from model $(2.2)$ with $\nu_{i}=\nu \exp \left\{X_{i 1} \gamma_{1}+X_{i 2} \gamma_{2}+X_{i 3} \gamma_{3}\right\}$ and $X_{i 3} \sim B(1,0.2)$. We chose $\gamma_{3}=-0.4$ and generated all of the other variables in the same way as in Simulation $A$. We analyzed the data by assuming study units are spatially correlated with the full set of covariates, i.e. $X_{1}, X_{2}$ and $X_{3}$, and the partial set of covariates, i.e. $X_{1}, X_{2}$. Table S11 in the Supplementary Materials presents a summary of estimates. When only $X_{1}$ and $X_{2}$ are included in the model, the estimates for $\gamma_{1}, \gamma_{2}$ are still close to the true values. We can also identify $X_{1}$ and $X_{2}$ as significant predictors for the duration from the estimates for $\beta_{1}$ and $\beta_{2}$. These results suggest that the missing covariate doesn't change the effects of other covariates on the longitudinal process and the duration.

Scenario (C.2) generated spatially correlated units with a covariate-dependent correlation function. We first simulated $Z_{i} \sim B(1,0.8)$ and considered the longitudinal model with $\nu_{i}=\nu \exp \left\{X_{i 1} \gamma_{1}+X_{i 2} \gamma_{2}\right\}$, i.e. $\psi_{2}=0, \rho_{2}=0$. Here, $\boldsymbol{\delta}_{1}=\left(\delta_{11}, \cdots, \delta_{1 n}\right)$ were generated from $\operatorname{MVN}\left(0, \Sigma_{1}^{*}\right)$ with the $(i, j)^{\text {th }}$ element in $\Sigma_{1}^{*}$ being $\psi_{1}^{2} \exp \left\{-\left|\boldsymbol{\omega}_{\boldsymbol{i}}-\boldsymbol{\omega}_{\boldsymbol{j}}\right| /\left(\rho\left(Z_{i}\right) \rho\left(Z_{j}\right)\right)\right.$ and $\operatorname{logit}\left[\rho\left(Z_{i}\right)\right]=\log (49)-3 Z_{i}$. We chose $\psi_{1}=0.25$ and analyzed the correlated data with the spatial correlation structure given in (2.3) and with the true spatial correlation function $\Sigma_{1}^{*}$. To make a comparison, we also approximate the value of $\rho_{1}$ in the mis-specified correlation function given in $(2.3)$ by $\rho_{1}=\mathrm{E}\left[\rho\left(Z_{i}\right) \rho\left(Z_{j}\right)\right]$. Table $\mathrm{S} 12$ in the Supplementary Materials summarized the estimates with the simulated data. With the true spatial 
correlation function, we assumed the coefficients associated with $\rho(\cdot)$ are known and only estimated $\psi_{1}$. Although the mis-specified correlation structure leads to biased estimates for parameters $\psi_{1}$ and $\rho_{1}$, we observe that the sample means of estimates for $\nu, \gamma$ are close to the true values. The estimates for $\beta$ with this mis-specified structure are also similar to the ones with the true correlation function and close to the estimates with the complete ISA duration. These results suggest that the reported analysis of the real-data can still be meaningful and interpretable even if an important covariate is missing in the correlation structure.

\section{Final Remarks}

We have considered the regression analysis of event durations with missing origins under the semiparametric AFT model. This is an extension of the work by Xiong, Braun, and $\mathrm{Hu}(2021)$ to the regression setting. Motivated by records of wildfires, we also extended the procedure to account for spatial correlation. We used our approach to estimate the distribution of the ISA duration given a set of covariates for lightning-caused wildfires, and we validated our results via a simulation study. Both the real-data analysis and the simulation studies indicate that inference based on conventional approaches could give misleading results. This confirms the importance of dealing with the missing time origins. The proof of the asymptotic properties of the estimators with spatially correlated units remains an open problem, but our simulation results appear to support the asymptotic validity of our approach.

Our approach not only evaluates the association of the ISA duration with the risk factors but also provides a distribution estimator. Given the covariates at the report time, our distribution estimator can be used to predict the full ISA duration. In the presence of spatial correlation, it can straightforwardly be extended to estimate the 
joint density, which will improve the deployment of wildfire suppression resources. In addition, although we assume the two random effects are independent with each other, our approach can be readily adapted by specifying the correlation between the two random effects.

Several future investigations would be worthwhile. First, our approach finds no association between the ISA duration and the covariates wind speed and fuel type that are believed to play important roles in the development of fires. This unexpected finding may be due to their strong association with the covariate region. A stratified data analysis may help to reveal the true effects of wind speed and fuel type. Although the application presented in this paper doesn't involve highdimensional data, it would be of both scientific and statistical interest to account for high-dimensional variable selection when applying this approach to other situations. Second, the environmental factors are often time-varying, and we could consider extending the model to dynamically predict the ISA duration. For example, we may consider linear transformation models and adapt our approach to approximate the start time and the inherent missing segment of the time-varying covariate. Further, the estimation based on spatially correlated data can be computationally intensive; feasible methods based on the composite likelihood (e.g. Paik and Ying, 2012) may reduce this burden. It is also worth branching out from the current spatial covariance functions to make it depend on directional variables. Following Neto et al. (2014), we can extend the proposed procedure to incorporate directional variables in the spatial covariance function. Finally, our approach is applicable to many practical situations with missing time origins. Examples include predicting the time from HIV infection to AIDS diagnosis (Doksum and Normand, 1995) and studying the gap times of the labour process for pregnant women with an unknown start time 
of the dilation process (Ma and Sundaram, 2018). A recent example is COVID-19 with asymptomatic infection and transmission. One can estimate the distribution of the incubation period with our approach by making use of the longitudinal viral load measures.

\section{Supplementary Materials}

The derivation of the asymptotic properties in Section 3.1.3, and additional tables and figures of the real data analysis in Section 4 and the simulation studies in Section 5 together with sample codes are provided in the online supplementary materials.

\section{Acknowledgements}

We are grateful to N. McLoughlin of Alberta Wildfire Management Branch for providing the wildland fire data. We thank G.A. Whitmore for his many constructive suggestions, and the $\mathrm{AE}$ and two referees for their careful reviews of a previous version of the manuscript. The research was supported by grants from the Natural Sciences and Engineering Research Council of Canada (NSERC) and a CRT grant from the Canadian Statistical Sciences Institute (CANSSI).

\section{References}

Banerjee, S., M. M. Wall, and B. P. Carlin (2003). Frailty modeling for spatially correlated survival data, with application to infant mortality in minnesota. Biostatistics 4(1), 123-142.

Buckley, J. and I. James (1979). Linear regression with censored data. Biometrika 66, 429-436.

Chhikara, R. S. and J. L. Folks (1989). The Inverse Gaussian Distribution: Theory, Methodology and Applications, Volume 162. New York, USA: Marcel Dekker, Inc.

Cumming, S. (2001). Forest type and wildfire in the alberta boreal mixedwood: what do fires burn? Ecological applications 11(1), 97-110.

Doksum, K. A. and S.-L. T. Normand (1995). Gaussian models for degradation processes-part i: Methods for the analysis of biomarker data. Lifetime Data Analysis 1(2), 131-144.

Forestry Canada Fire Danger Group (1992). Development and structure of the Canadian forest fire behavior prediction system, Volume 3. Forestry Canada. 
Furgal, A. K., A. Sen, and J. M. Taylor (2019). Review and comparison of computational approaches for joint longitudinal and time-to-event models. International Statistical Review 87(2), 393-418.

Goetghebeur, E. and L. Ryan (2000). Semiparametric regression analysis of intervalcensored data. Biometrics 56(4), 1139-1144.

Hastings, W. K. (1970). Monte carlo sampling methods using markov chains and their applications. Biometrika 57(1), 97-109.

Komárek, A. and M. A. Komárek (2015). Package 'smoothsurv'.

Lee, M.-L. T. and G. A. Whitmore (2006). Threshold regression for survival analysis: modeling event times by a stochastic process reaching a boundary. Statistical Science 21(4), 501-513.

Li, Y. and L. Ryan (2002). Modeling spatial survival data using semiparametric frailty models. Biometrics 58(2), 287-297.

Loader, C. R. (1999). Bandwidth selection: classical or plug-in? The Annals of Statistics 27(2), 415-438.

Ma, L. and R. Sundaram (2018). Analysis of gap times based on panel count data with informative observation times and unknown start time. Journal of the American Statistical Association 113(521), 294-305.

Martell, D. L. (2007). Forest fire management. In Handbook of Operations Research in Natural Resources, pp. 489-509. Springer.

Metropolis, N., A. W. Rosenbluth, M. N. Rosenbluth, A. H. Teller, and E. Teller (1953). Equation of state calculations by fast computing machines. The Journal of Chemical Physics 21(6), 1087-1092.

Morin, A. A. (2014). A spatial analysis of forest fire survival and a marked cluster process for simulating fire load. The University of Western Ontario, London, Ontario, Canada: MSc thesis.

Motarjem, K., M. Mohammadzadeh, and A. Abyar (2017). Geostatistical survival model with gaussian random effect. Statistical Papers, 1-23.

Neto, J. H. V., A. M. Schmidt, and P. Guttorp (2014). Accounting for spatially varying directional effects in spatial covariance structures. Journal of the Royal Statistical Society: Series C: Applied Statistics, 103-122.

Ning, J., J. Qin, and Y. Shen (2011). Buckley-james-type estimator with rightcensored and length-biased data. Biometrics 67(4), 1369-1378.

Paik, J. and Z. Ying (2012). A composite likelihood approach for spatially correlated survival data. Computational statistics \&f data analysis 56(1), 209-216.

Papageorgiou, G., K. Mauff, A. Tomer, and D. Rizopoulos (2019). An overview of joint modeling of time-to-event and longitudinal outcomes. Annual review of statistics and its application. 
Qin, J., C. You, Q. Lin, T. Hu, S. Yu, and X.-H. Zhou (2020). Estimation of incubation period distribution of covid-19 using disease onset forward time: a novel cross-sectional and forward follow-up study. Science advances 6(33), eabc1202.

Tremblay, P.-O., T. Duchesne, and S. G. Cumming (2018). Survival analysis and classification methods for forest fire size. PloS one 13(1).

Tseng, Y.-K., F. Hsieh, and J.-L. Wang (2005). Joint modelling of accelerated failure time and longitudinal data. Biometrika 92(3), 587-603.

Wu, L., W. Liu, and X. J. Hu (2010). Joint inference on hiv viral dynamics and immune suppression in presence of measurement errors. Biometrics 66 (2), 327335.

Wulfsohn, M. S. and A. A. Tsiatis (1997). A joint model for survival and longitudinal data measured with error. Biometrics, 330-339.

Xiong, Y., D. Bingham, W. J. Braun, and X. J. Hu (2019). Moran's i statistic-based nonparametric test with spatio-temporal observations. Journal of Nonparametric Statistics 31(1), 244-267.

Xiong, Y., W. J. Braun, and X. J. Hu (2021). Estimating duration distribution aided by auxiliary longitudinal measures in presence of missing time origin. Lifetime Data Analysis. published online.

Department of Statistics and Actuarial Science, Simon Fraser University

E-mail: (yi_xiong@sfu.ca)

Department of Computer Science, Mathematics, Physics and Statistics, University of British Columbia-Okanagan

E-mail: (john.braun@ubc.ca)

Département de mathématiques et de statistique, Université Laval

E-mail: (thierry.duchesne@mat.ulaval.ca)

Department of Statistics and Actuarial Science, Simon Fraser University

E-mail: (joanh@stat.sfu.ca) 
Table 1: Estimates of Parameters in the Longitudinal Model (2.2)

\begin{tabular}{|c|c|c|c|c|c|c|c|c|c|c|c|c|}
\hline & & $\nu$ & $\sigma$ & $\psi_{1}^{2}$ & $\rho_{1}$ & $\psi_{2}^{2}$ & $\rho_{2}$ & $\gamma_{\text {Region:upper }}^{\S}$ & $\gamma_{W \text { indSpeed }}$ & $\gamma_{\text {Fuel:C1 }}^{\S}$ & $\gamma_{\text {Fuel:C2 }}^{\S}$ & $\gamma_{\text {Fuel:M2 }}^{\S}$ \\
\hline \multirow{4}{*}{$\begin{array}{l}\text { Random } \\
\text { Intercept }\end{array}$} & \multicolumn{5}{|c|}{ Independent Units } & & & & & \multirow{4}{*}{$\begin{array}{l}0.528 \\
0.399 \\
\end{array}$} & \multirow{4}{*}{$\begin{array}{l}0.252 \\
0.309\end{array}$} & \multirow{4}{*}{$\begin{array}{r}-0.370 \\
0.434 \\
\end{array}$} \\
\hline & Est. & 0.036 & 1.272 & 0.020 & & & & 0.225 & 2.878 & & & \\
\hline & $\mathrm{SE}$ & 0.024 & 0.026 & 0.011 & & & & 0.173 & 0.521 & & & \\
\hline & $\mathrm{AIC}$ & 2887.7 & & & & & & & & & & \\
\hline \multirow{3}{*}{$\begin{array}{l}\text { Mixed } \\
\text { Effect }\end{array}$} & Est. & 0.044 & 1.270 & 0.008 & & 0.011 & & 0.378 & 3.231 & 0.300 & 0.417 & -0.025 \\
\hline & $\mathrm{SE}$ & 0.022 & 0.026 & 0.003 & & 0.005 & & 0.148 & 0.507 & 0.435 & 0.331 & 0.462 \\
\hline & $\mathrm{AIC}$ & 1343.6 & & & & & & \multicolumn{5}{|c|}{ 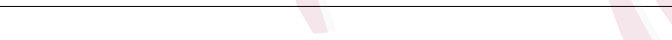 } \\
\hline \multirow{4}{*}{$\begin{array}{l}\text { Random } \\
\text { Intercept }\end{array}$} & \multicolumn{5}{|c|}{ Correlated Units } & & & & & & \multirow{4}{*}{$\begin{array}{l}0.065 \\
0.298 \\
\end{array}$} & \\
\hline & Est. & 0.041 & 1.272 & 0.015 & 0.017 & & & 0.294 & 2.830 & 0.536 & & 0.306 \\
\hline & $\mathrm{SE}$ & 0.025 & 0.026 & 0.007 & 0.008 & & & 0.141 & 0.422 & 0.274 & & 0.424 \\
\hline & $\mathrm{AIC}$ & 1353.6 & & & & & & & & & & thes \\
\hline Mixed & Est. & 0.043 & 1.272 & 0.019 & 2.927 & 0.010 & 0.011 & 0.786 & 3.844 & -0.006 & 0.138 & -0.751 \\
\hline Effect & $\mathrm{SE}$ & 0.024 & 0.026 & 0.006 & 0.038 & 0.005 & 0.006 & 0.133 & 0.479 & 0.426 & 0.316 & 0.346 \\
\hline
\end{tabular}

$\boldsymbol{\gamma}_{\text {Region:upper }}^{\S}$ : coefficient for categorical variable Region and baseline category is lower region.

$\boldsymbol{\gamma}_{\text {Fuel:C1 }}^{\S}, \boldsymbol{\gamma}_{\text {Fuel:C2 }}^{\S}, \boldsymbol{\gamma}_{\text {Fuel:M2 }}^{\S}$ : coefficients for categorical variable Fuel type and baseline category is other fuel types.

Table 2: Estimates of Parameters in Models LRM-Indpt 2.4a and LRM-Crrltd (2.4c)

\begin{tabular}{|c|c|c|c|c|c|c|c|c|}
\hline & $\beta_{0}$ & $\beta_{\text {Region:upper }}^{\S}$ & $\beta_{\text {WindSpeed }}$ & $\beta_{\text {Fuel:C1 }}^{\S}$ & $\beta_{\text {Fuel:C2 }}^{\S}$ & $\beta_{\text {Fuel:M2 }}^{\S}$ & $\beta_{\delta_{1}}$ & $\beta_{\delta_{2}}$ \\
\hline & \multicolumn{4}{|c|}{ Independent Units } & & & & \\
\hline \multirow{2}{*}{$\begin{array}{l}\text { Observed } \\
\text { duration }\end{array}$} & Est. $\quad 0.014$ & -0.097 & -0.405 & -0.499 & -0.341 & -0.186 & & \\
\hline & 0.281 & 0.147 & 0.521 & 0.372 & 0.267 & 0.327 & & \\
\hline \multirow{4}{*}{$\begin{array}{l}\text { Interval- } \\
\text {-censored }\end{array}$} & Est. 2.013 & 0.092 & -0.437 & -0.030 & -0.001 & 0.012 & & \\
\hline & 0.199 & 0.109 & 0.447 & 0.261 & 0.181 & 0.235 & & \\
\hline & Est. 3.446 & 0.041 & 0.211 & 0.026 & -0.023 & -0.260 & & \\
\hline & 0.101 & 0.063 & 0.187 & 0.131 & 0.092 & 0.243 & & \\
\hline Proposed Random & Est. 0.981 & 0.341 & -0.230 & -0.231 & -0.097 & -0.446 & & \\
\hline intercept & 0.323 & 0.175 & 0.600 & 0.425 & 0.308 & 0.377 & & \\
\hline Mixed & Est. $\quad 0.967$ & 0.292 & -0.287 & -0.183 & -0.046 & -0.406 & & \\
\hline Effect & 0.325 & 0.124 & 0.612 & 0.434 & 0.306 & 0.379 & & \\
\hline & \multicolumn{4}{|c|}{ Correlated Units } & & & & \\
\hline \multirow{2}{*}{$\begin{array}{l}\text { Observed } \\
\text { duration }\end{array}$} & Est. $\quad 0.012$ & -0.112 & -0.344 & -0.520 & -0.349 & -0.166 & -0.978 & -0.514 \\
\hline & 0.281 & 0.147 & 0.523 & 0.372 & 0.267 & 0.327 & 0.734 & 0.709 \\
\hline$R_{\max }=12$ & Est. 2.026 & 0.087 & -0.465 & -0.037 & 0.001 & 0.007 & -0.214 & 0.066 \\
\hline \multirow{3}{*}{$\begin{array}{l}\text { Interval- } \\
\text {-censored }\end{array}$} & 0.201 & 0.105 & 0.433 & 0.254 & 0.184 & 0.237 & 0.574 & 0.532 \\
\hline & Est. 3.445 & 0.043 & 0.208 & 0.030 & -0.022 & -0.261 & 0.033 & -0.168 \\
\hline & 0.102 & 0.060 & 0.191 & 0.125 & 0.088 & 0.238 & 0.330 & 0.305 \\
\hline \multirow[t]{4}{*}{ Proposed } & Est. 0.987 & 0.382 & -0.294 & -0.238 & -0.076 & -0.446 & -0.486 & \\
\hline & 0.322 & 0.172 & 0.602 & 0.446 & 0.332 & 0.383 & 0.575 & \\
\hline & Est. 0.880 & 0.387 & -0.248 & -0.087 & 0.053 & -0.256 & -0.862 & -0.603 \\
\hline & 0.319 & 0.179 & 0.600 & 0.417 & 0.317 & 0.376 & 0.339 & 0.371 \\
\hline
\end{tabular}

$\boldsymbol{\beta}_{\text {Region:upper }}^{\S}$ : regression coefficient for categorical variable Region and baseline category is lower region.

$\boldsymbol{\beta}_{F u e l: C 1}^{\S}, \boldsymbol{\beta}_{F u e l: C 2}^{\S}, \boldsymbol{\beta}_{F u e l: M 2}^{\S}$ : regression coefficients for categorical variable Fuel type and baseline category is other fuel types. 
Table 3: Estimates of Parameters in Models PLRM-Indpt (2.4b) and PLRM-Crrltd (2.4d, with $h_{0}$ estimated via kernel smoothing

\begin{tabular}{|c|c|c|c|c|c|c|c|c|}
\hline & & $\beta_{0}$ & $\beta_{\text {Region:Northeast }}^{\dagger}$ & $\beta_{\text {Fuel:C1 }}^{\dagger}$ & $\beta_{\text {Fuel:C2 }}^{\dagger}$ & $\beta_{\text {Fuel:M2 }}^{\dagger}$ & $\beta_{\delta_{1}}^{\dagger}$ & $\beta_{\delta_{2}}^{\dagger}$ \\
\hline & \multicolumn{4}{|c|}{ Model 2.4b], Independent Units } & & & & \\
\hline Random & Est. & 0.929 & 0.382 & -0.231 & -0.097 & -0.435 & & \\
\hline Intercept & SE & 0.325 & 0.193 & 0.471 & 0.341 & 0.416 & & \\
\hline Mixed & Est. & 0.902 & 0.393 & -0.182 & -0.046 & -0.393 & & \\
\hline Effect & $\mathrm{SE}$ & 0.327 & 0.192 & 0.479 & 0.339 & 0.418 & & \\
\hline & \multicolumn{4}{|c|}{ Model [2.4d], Correlated Units } & & & & \\
\hline Random & Est. & 0.920 & 0.383 & -0.238 & -0.076 & -0.432 & -0.477 & \\
\hline Intercept & SE & 0.328 & 0.188 & 0.468 & 0.352 & 0.402 & 0.534 & \\
\hline Mixed & Est. & 0.924 & 0.386 & -0.172 & -0.071 & -0.409 & -0.848 & -0.540 \\
\hline Effect & SE & 0.329 & 0.189 & 0.475 & 0.335 & 0.382 & 0.433 & 0.557 \\
\hline
\end{tabular}

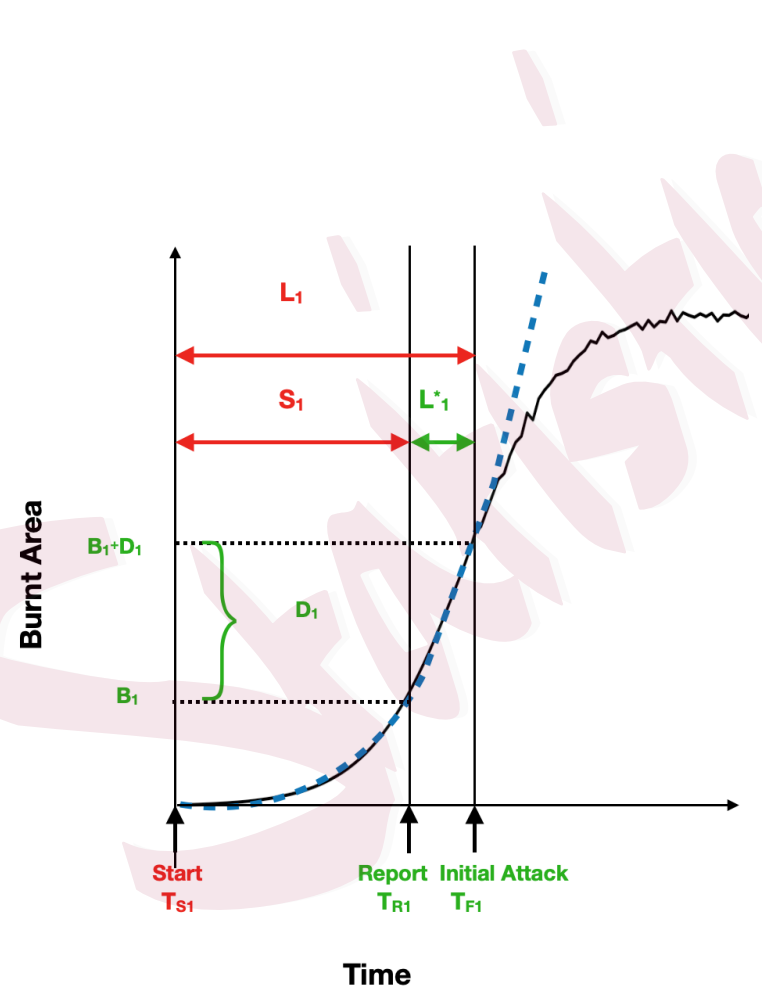

Fire 1

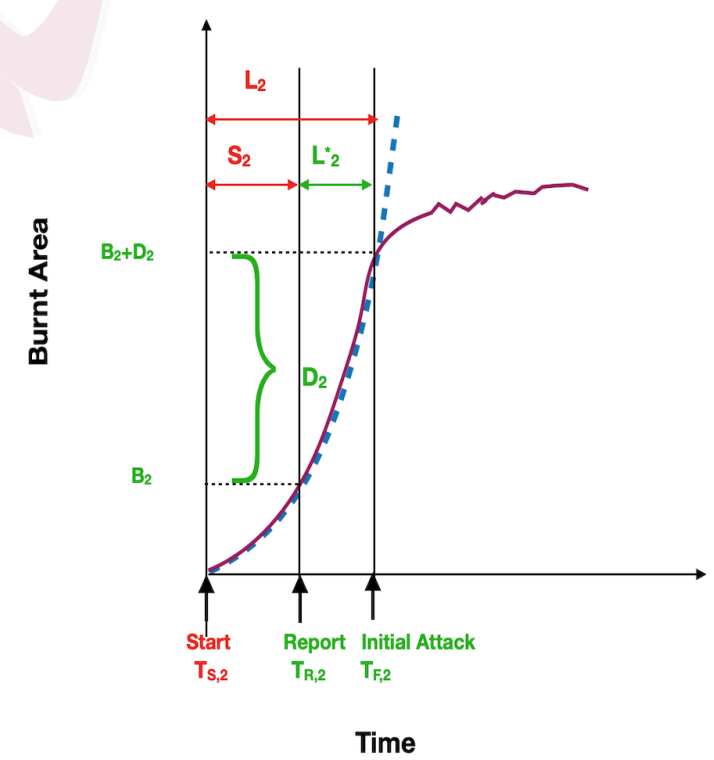

Fire 2

Figure 1: Hypothetical description of progression through fire management phases for two fires with different covariate values 
Est. Distribution of Fires in Lower Region, M2 Fuel Type

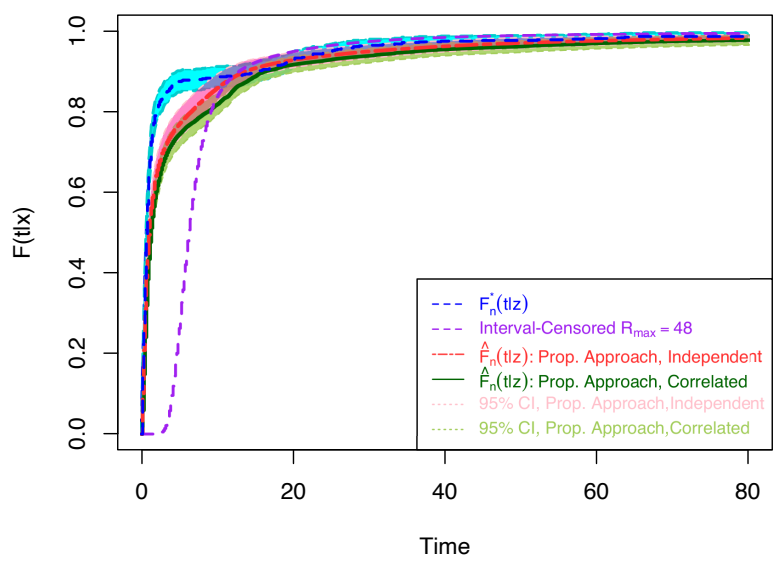

(a.1) Lower region, M2 type, wind speed $=7.75 \mathrm{~km} / \mathrm{h}$.

Est. Distribution of Fires in Lower Region, C1 Fuel Type

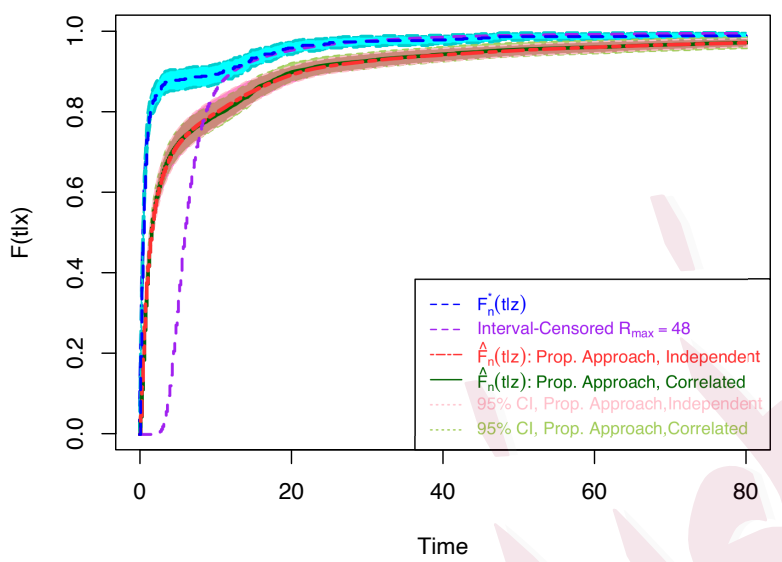

(b.1) Lower region, C1 type, wind speed $=7.75 \mathrm{~km} / \mathrm{h}$.

Est. Distribution of Fires in Lower Region, Other Fuel Type

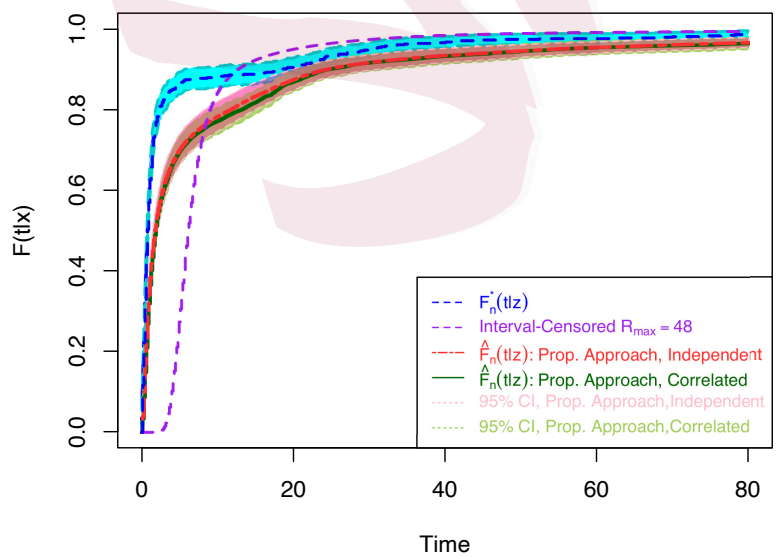

(c.1) Lower region, Other type, wind speed $=7.75 \mathrm{~km} / \mathrm{h}$.
Est. Distribution of Fires in Upper Region, M2 Fuel Type

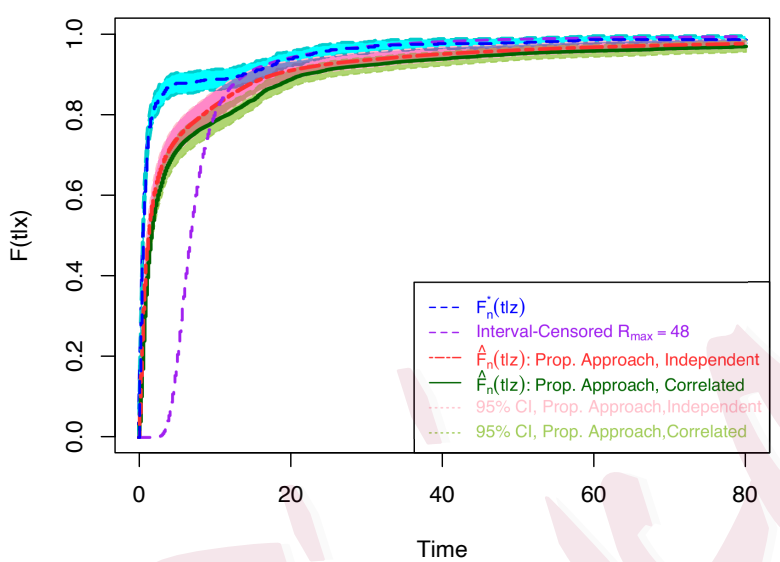

(a.2) Upper region, M2 type, wind speed $=7.75 \mathrm{~km} / \mathrm{h}$.

Est. Distribution of Fires in Upper Region, C1 Fuel Type

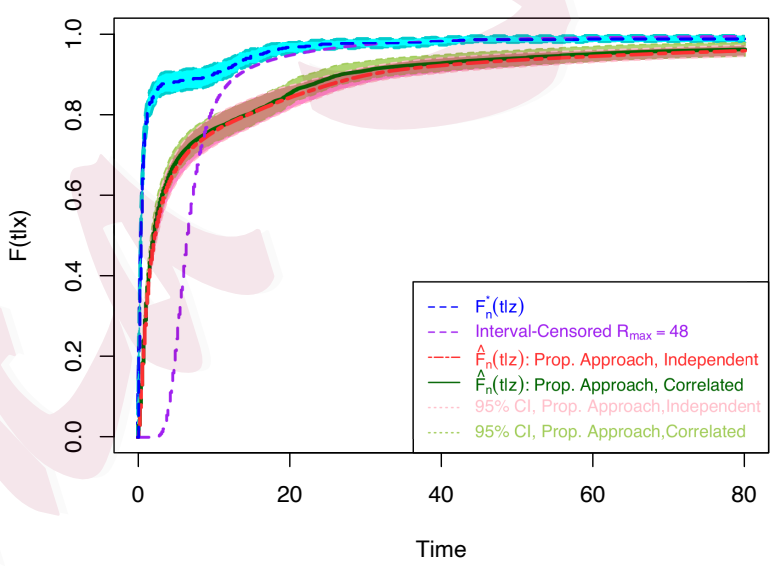

(b.2) Upper region, C1 type, wind speed $=7.75 \mathrm{~km} / \mathrm{h}$.

Est. Distribution of Fires in Upper Region, Other Fuel Type

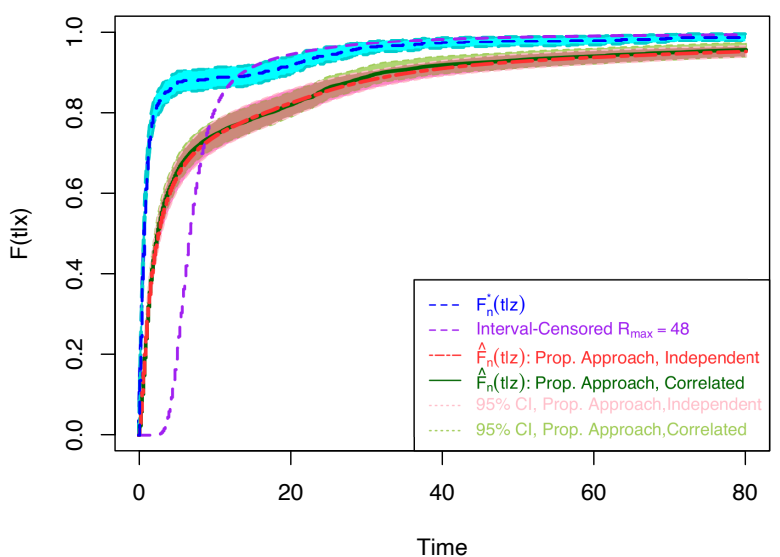

(c.2) Upper region, Other type, wind speed $=7.75 \mathrm{~km} / \mathrm{h}$.

Figure 2: Estimated $F(t \mid \mathbf{x})$. 
REFERENCES

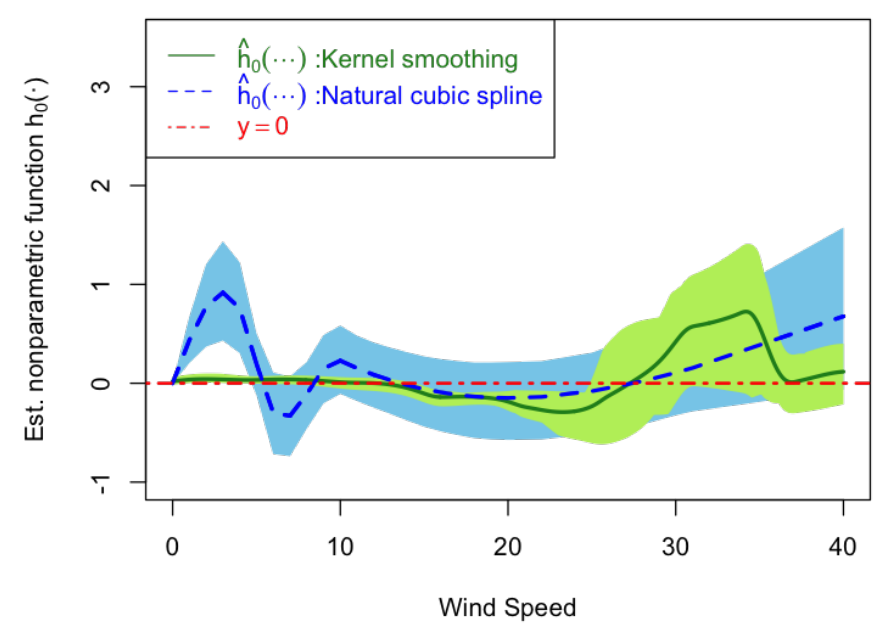

(a) Model PLRM-Indpt 2.4b

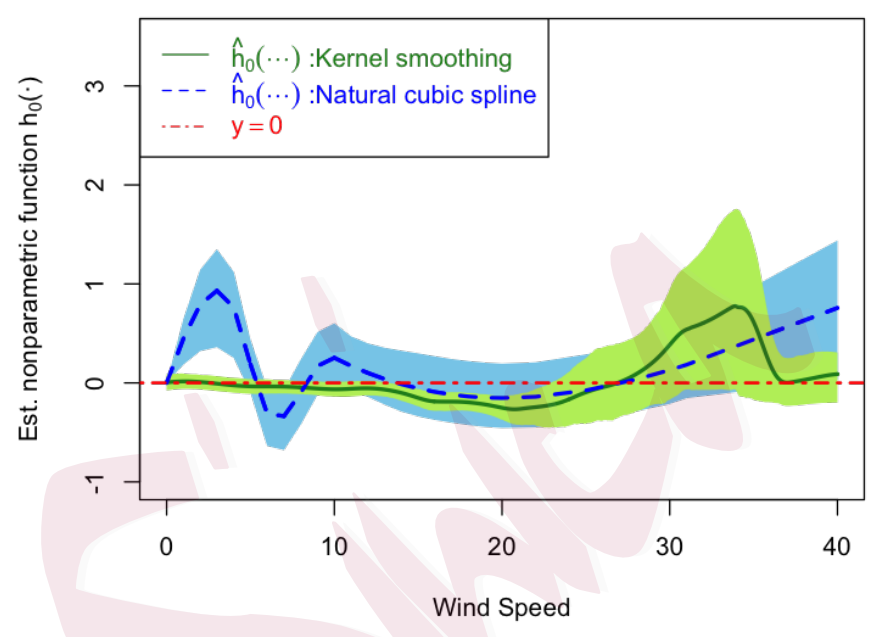

(b) Model PLRM-Crrltd 2.4d

Figure 3: Plots of estimated nonparametric function $h_{0}(\cdot)$ of wind speed. The shaded area represents the $95 \%$ pointwise confidence intervals, which are approximated by the $2.5 \%$ and $97.5 \%$ quantiles of realizations of $\hat{h}_{0}(\cdot)$ using the bootstrap with resample size 1000 .

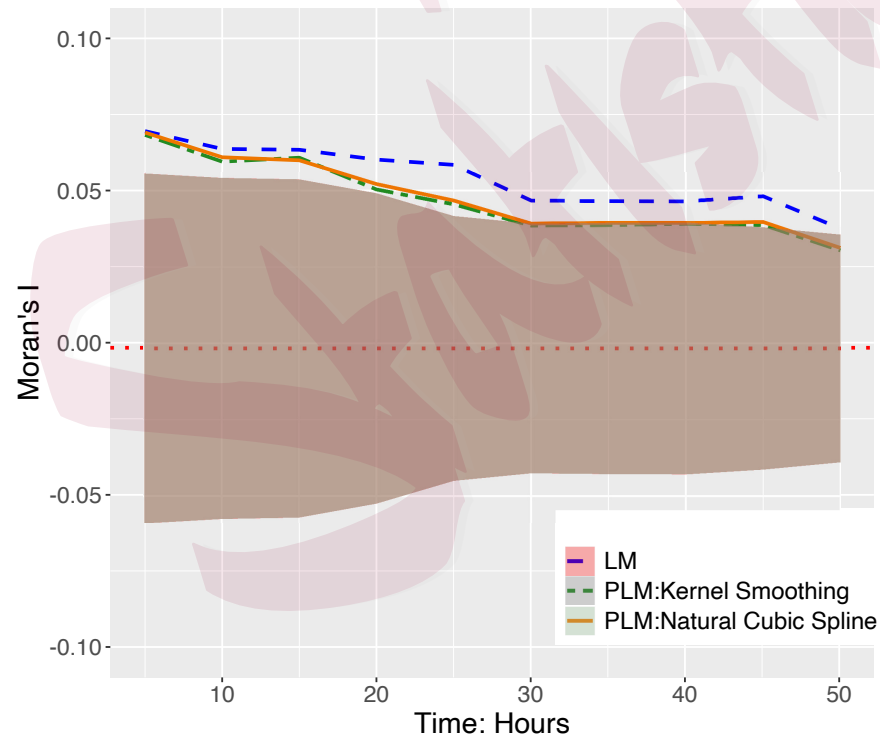

(a) Models LRM-Indpt 2.4a and PLRM-Indpt (2.4b), with independent units

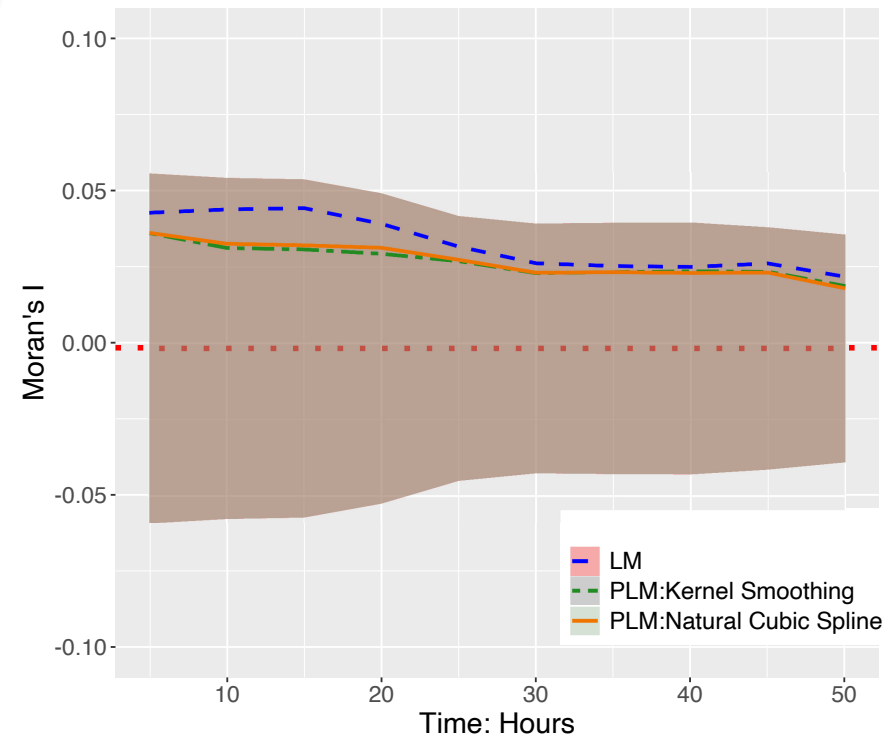

(b) Models LRM-Crrltd 2.4c) and PLRM-Crrltd $(2.4 \mathrm{~d})$, with correlated units

Figure 4: Model diagnosis by Moran's I using residuals from different regression models. 